\title{
Observation and modelling of main-sequence star chromospheres
}

\section{Two-component model chromospheres for nine M1 dwarfs ${ }^{\star}$}

\author{
E. R. Houdebine
}

\begin{abstract}
25 rue du Dr. Laulaigne, 49670 Valanjou, France
\end{abstract}
e-mail: eric_houdebine@yahoo.fr

Received 30 December 2008 / Accepted 21 April 2009

\begin{abstract}
Aims. We aim to constrain the $\mathrm{H}_{\alpha}$, CaII H and CaII K line profiles of quiescent and active regions of nine dM1 stars of near solar metallicity: Gl 2, GJ 1010A, Gl 49, Gl 150.1B, Gl 205, Gl 229, Gl 526, G192-11A, and Gl 880.

Methods. We propose a new method for building two-component model chromospheres for dM1 stars-based on simple constraints and a grid of model atmospheres developed by Houdebine \& Stempels. This method is based on the measurements of the equivalent width of $\mathrm{H}_{\alpha}$ and CaII $\mathrm{H} \& \mathrm{~K}$. Based on the peculiar relationship between these two equivalent widths in the model atmospheres, our solutions provide an exact match of these equivalent widths.

Results. We obtain two component (quiescent and active region) model chromospheres for our nine target stars. We fit the $\mathrm{H}_{\alpha}$, $\mathrm{CaII} \mathrm{H}$, and CaII K profiles for these stars. These models show that seven of these stars lie in the intermediate activity range between $\mathrm{H}_{\alpha}$ maximum absorption and emission. Two stars (Gl 49 and G192-11A) are quite active with $\mathrm{H}_{\alpha}$ emission profiles in plages. As far as the CaII emission is concerned, these two stars are almost as active as dM1e stars. Two stars (GJ 1010A and Gl 526) have lower activity levels with narrower and weaker $\mathrm{H}_{\alpha}$ profiles. The range of activity covered by these stars is a factor of 13 in the CaII lines, from low activity to activity levels almost as high as those of dM1e stars.

Our method sometimes provides two solutions of the observed $\mathrm{H}_{\alpha}$ equivalent width as a function of the quiescent region $\mathrm{H}_{\alpha}$ equivalent width. For G1 205, one of the solutions is shown to be impossible for the assumptions that we use. For Gl 49 and G192-11A, two solutions are possible; a low solution (low CaII $E W$ ) and a high solution (high CaII $E W$ ). The difference between these two solutions is mainly in the plage-filling factor. The two solutions give almost identical $\mathrm{H}_{\alpha}$ and CaII profiles. We prefer the low solutions because the filling factors are in closer agreement with those of other stars. We find plage-filling factors typically in the range $20 \%-40 \%$. We also find that it is the chromospheric pressure rather than the filling factor that increases with increasing activity.

We define a minimum theoretical $\mathrm{H}_{\alpha}$ equivalent width as a function of the mean CaII $\mathrm{H} \& \mathrm{~K}$ equivalent width. We show that our observations agree well with this lower limit. We also show that the properties of the chromosphere in quiescent and active regions correlate with the mean CaII H \& K equivalent width. This could be useful in future studies to derive an estimate of the chromospheric properties from the observed mean CaII H \& K equivalent width.
\end{abstract}

Key words. line: formation - radiative transfer - stars: activity - stars: low-mass - stars: chromospheres stars: late-type

\section{Introduction}

The chromospheres of two types of dM stars have been modelled in previous studies: active dMe stars (e.g., Cram \& Mullan 1979; Kelch et al. 1979; Giampapa et al. 1981; Houdebine \& Doyle 1994a,b; Mauas \& Falchi 1994; Lanzafame \& Byrne 1995; Houdebine \& Stempels 1997; Short et al. 1997; Short \& Doyle 1998a,b; Jevremovic et al. 2000; Mauas 2000) and very inactive (basal) dM stars (e.g. Doyle et al. 1994; Houdebine \& Doyle 1995b). Only a few stars of intermediate activity have been modelled so far (Walkowicz 2008). This is probably because two-component model chromospheres (quiescent and active regions) are necessary to describe those stars, with each component making a significant contribution to the total profiles. Here, we present two-component model chromospheres for these intermediate-activity dM1 stars.

Uniform model chromospheres were systematically used to reproduce the spectra of $\mathrm{dMe}$ stars. The contribution from the

^ Based on observations collected at Observatoire de Haute Provence, France quiescent areas were not taken into account. Most model chromospheres of dMe stars (e.g., Cram \& Mullan 1979; Kelch et al. 1979; Giampapa et al. 1981; Houdebine \& Doyle 1994a,b; Mauas \& Falchi 1994; Lanzafame \& Byrne 1995; Houdebine \& Stempels 1997; Short et al. 1997; Short \& Doyle 1998a,b; Jevremovic et al. 2000; Mauas 2000) indeed model the entire chromospheric line profiles of the stars. Houdebine (2009a, Paper X) showed that if one wants to understand the chromosphere of these stars, one must take account of the contributions from quiescent regions. Its contribution to the copmplete $\mathrm{H}_{\alpha}$ profile is not negligible, in particular for the central self-reversal. The contribution to the CaII lines should also be taken into account. Similarly, the comparison of grids of models with observations show that the models cannot reproduce the observations accurately and that a one-component model chromosphere is incorrect (Houdebine 1995, 1996; Houdebine \& Stempels 1997).

One must ask whether there are any plages on dM1 stars or the chromospheric emission originates from the network? All dM1 stars that we observed (main-sequence dwarfs, not including subdwarfs) are more active than the Sun per unit surface area (Paper VI). The sun has plages, and we may therefore 
expect dM1 stars to have plages. However, the time-averaged spectrum of the Sun is not dominated by plages, which is one additional difference with dM1e stars. Is this also true for dM1 stars? Rotational modulation was detected in slow rotators from dK2 to dK7 (Vaughan et al. 1981; Baliunas et al. 1983). It is therefore probable that close dM1 stars have plages. Many dM1 stars also have filled in $\mathrm{H}_{\alpha}$ profiles and are almost as active as dM1e stars. These stars therefore, probably have extended plages on their surface. Hence, two-component modelling of their chromospheres appears the most accurate approach.

In the present study, we investigate the chromosphere of solar-metallicity dM1 stars. According to the radius-metallicity relation found by Houdebine (2008, Paper VII), these stars have a relatively large radius. Furthermore, the radius of a dM1 star is correlated with magnetic activity (Houdebine \& Stempels 1997, Paper VI). Therefore, the stars that we investigate here have relatively high levels of magnetic activity, compared to other smaller metal-poor M1 dwarfs. Hence, our targets do not have basal chromospheres; they have intermediate-activity chromospheres often with filled-in $\mathrm{H}_{\alpha}$ profiles. We see below that two of our targets have activity levels close to those of dM1e stars.

In Paper X, we modelled the spectrum of Gl 205, which is an intermediate-activity star (filled-in $\mathrm{H}_{\alpha}$ ). By simple considerations and comparison with the model grid of Houdebine \& Stempels (1997), we derived a two-component model chromosphere for this star. Here, we formalize this approach and find a method for deriving two-component model chromospheres for any dM1 star, based on the grid of model atmospheres of Houdebine \& Stempels (1997, Paper VI). This model grid assumes solar metallicity, which is why we consider only nearsolar-metallicity M1 dwarfs. This modelling is based mostly on simultaneous high-resolution observations of the $\mathrm{H}_{\alpha}$ line and the CaII H \& K lines.

\section{Observations and data reduction}

The observations presented here were acquired at the $1.93 \mathrm{~m}$ telescope of Observatoire de Haute Provence, with the ELODIE spectrograph. ELODIE is a cross-dispersed echelle spectrograph with an original optical design that yields evenly spaced orders (see Special Issue of "La Lettre de l'OHP", 1995). The principal objective in building this spectrograph was to detect exoplanets. It was therefore designed to be very stable in wavelength. The spectral coverage is from $3890 \AA$ to $6820 \AA$ with a resolving power of about 45000 . The cross correlation profiles used to measure the $v \sin i$ in Paper VII show that for stars with $v \sin i$ less than $1 \mathrm{~km} \mathrm{~s}^{-1}$, the mean $F W H M$ is $0.197 \AA$, indicating a resolving power greater than or equal to 29000 . The $F W H M$ measurements of the Thorium-Argon spectral lines indicate a resolving power of 30000 . The resolving power therefore seems slightly less than initially claimed. For the convolution of the theoretical spectra, we adopt below a resolving power of 30000 which provides closer agreement with observations.

For most of the data, we used an automatic reduction procedure made available by Queloz (1994). The scattered interorder light was then extracted and fitted. The in-order scattered light was interpolated from the inter-order fitted light. The inorder scattered light was then subtracted to the order light. The scattered light is important in the blue but rapidly becomes negligible in the red. Different orders of the echelle spectra were merged by averaging and scaling overlapping wavelength domains. In the overlapping domains, successive orders were in good agreement with scaling differences of the order of $10 \%$.
Table 1. Stellar properties and spectral line equivalent widths.

\begin{tabular}{cccccccccc}
\hline \hline Star & $\begin{array}{c}\text { Radius }[\mathrm{M} / \mathrm{H}] \\
\left(R_{\odot}\right)\end{array}$ & $\begin{array}{c}T_{\text {eff }} \\
(\mathrm{dex})\end{array}$ & $\begin{array}{c}v \sin i \\
(\mathrm{~K})\end{array}$ & $\begin{array}{c}\mathrm{H}_{\alpha} E W \\
\left.\mathrm{~km} \mathrm{~s}^{-1}\right)\end{array}$ & $\begin{array}{c} \pm \sigma \\
(\AA)\end{array}$ & $\begin{array}{c}\text { CaII } E W \\
(\AA)\end{array}$ & $\begin{array}{c}(\AA) \\
(\AA)\end{array}$ & $(\AA)$ \\
\hline G1 2 & 0.618 & -0.018 & 3480 & $(1.2)$ & 0.353 & 0.018 & -1.57 & 0.27 \\
GJ 1010A & 0.576 & -0.098 & 3450 & - & 0.371 & 0.022 & -0.61 & 0.27 \\
G1 49 & 0.657 & 0.026 & 3450 & $(1.4)$ & 0.254 & 0.019 & -2.38 & 0.13 \\
G1 150.1B & 0.583 & -0.084 & 3480 & 2.2 & 0.439 & 0.023 & $(-1.4)$ & - \\
G1 205 & 0.758 & 0.101 & 3493 & $(1.7)$ & 0.459 & 0.015 & -1.43 & 0.31 \\
G1 229 & 0.695 & 0.060 & 3520 & $(1.3)$ & 0.497 & 0.017 & -1.01 & 0.1 \\
G1 526 & 0.582 & -0.0863470 & - & 0.463 & 0.020 & -0.491 & 0.1 \\
G192-11A & 0.626 & -0.009 & 3510 & $(1.4)$ & 0.249 & 0.010 & -2.41 & 0.16 \\
G1 880 & 0.669 & 0.040 & 3470 & $(1.3)$ & 0.465 & 0.022 & -1.19 & 0.37 \\
\hline
\end{tabular}

To isolate stars with close photospheric structures and effective temperatures, our stars were selected according to their $R-I$ infrared color, which is an appropriate effective temperature indicator (Legget 1992). We selected stars with $(R-I) \mathrm{c}$ in the range [1.064;1.196] (Papers VI and VII). Among the stars that we observed, we found eight stars of near-solar metallicity (within the measurement errors): Gl2, GJ 1010A, Gl 49, Gl 150.1B, Gl 205, Gl 229, G192-11A, and Gl 880. Gl 526 was not observed in our program, but it is also a near-solar metallicity M1 dwarf. In this paper, we used the spectral-line measurements compiled in Papers VI (for $\mathrm{H}_{\alpha}$ ) and IX (for the CaII lines) for G1526. We summarize the physical properties of these stars in Table 1 (from Paper VII). In this table we also provide the mean equivalent width of the CaII $\mathrm{H} \& \mathrm{~K}$ lines and the equivalent width of $\mathrm{H}_{\alpha}$ measured from our ELODIE spectra (Paper VI) or from the literature for G1526. We note that these stars do not have homogeneous activity levels. The level of activity is rather high for G1 49 and G192-11A, but significantly lower for GJ 1010A and G1526. We note that our ELODIE spectra are of a rather low $\mathrm{S} / \mathrm{N}$ ratio about the CaII lines, which infers relatively large error domains for our observations (see Paper VI). For $\mathrm{Gl} 229$, the $\mathrm{S} / \mathrm{N}$ ratio is too low to measure the equivalent widths of the CaII lines. For this star, we used the measurements compiled in Paper VI. Gl 205 was studied in Paper X, but we reinvestigate the chromosphere of this star in the light of the new method that we derive below.

The $\mathrm{S} / \mathrm{N}$ ratio of our observations close to the $\mathrm{H}_{\alpha}$ line is higher, but a large uncertainty remains in the measurements of the equivalent widths because of the difficulty in determining the continuum level in $\mathrm{M}$ dwarfs, and blends in the $\mathrm{H}_{\alpha}$ profile. The method used to measure the equivalent widths was described in Houdebine \& Stempels (1997, Paper VI).

\section{Constraining quiescent and active regions}

To progress in semi-empirical modelling of $\mathrm{M}$ dwarf chromospheres, we must consider models with two-component chromospheres. We discuss below the constraints attainable from presently available observations.

\subsection{High-contrast spectral lines}

Some spectral lines may have particularly high contrast between active and quiescent regions. This is notably the case for the HeI $\lambda 5876$ and the HeI $\lambda 10830$ lines in the Sun. In solar plages, they appear in absorption and are either not detectable or in the quiet Sun, very weak, (e.g., Andretta \& Giampapa 1995). Andretta \& Giampapa (1995) used these two spectral features to derive filling factors for the active regions for a number of 
$\mathrm{F}$ and $\mathrm{G}$ type stars. They obtained filling factors in the range $1 \%-62 \%$. Unfortunately, calculations for $\mathrm{M}$ dwarfs are not currently available and we cannot use this method here. However, we note a high contrast in the HeI $\lambda 5876$ line between Gl 205 and the active star G1867A (Paper X). In the former star, this line is not detected, whereas in the latter star it reveals strong emission, probably originating in plages. This line probably has a high contrast between quiescent and active regions on $\mathrm{dMe}$ stars. Models of this line could be used to constrain the activeregion filling factors.

However, there is one major problem with these Helium emission lines. It is well known that HeI and HeII lines exhibit an excess of emission in the quiet Sun with respect to other spectral lines from the transition region (e.g., Jordan 1975). These excesses attain of factors as high as $\sim 15$ and $\sim 6-8$ in the resonance lines of HeI and HeII, respectively. The possible enhancement mechanisms are of three types: (i) turbulent motions (e.g; Andretta et al. 2000), (ii) non-thermal electrons (e.g., Smith 2003), and, (iii) diffusion of hydrogen and helium (e.g., Fontenla et al. 2002). Some discussion of these mechanisms can be found in Jordan et al. (2005). These mechanisms are also likely to occur in $\mathrm{M}$ dwarfs, which implies that it is difficult to model HeI lines, and hence derive from them correct filling factors. Nevertheless, Lanzafame \& Byrne (1995) found that they could reproduce their HeI observations of Gl 182 with a simple model compatible with other observational constraints. They also found that EUV photoionization and streaming particles are of negligible effect in late-type stars. These findings are interesting and need to be confirmed, although the Helium spectral diagnostics at this stage must be interpreted with great care.

Sim \& Jordan (2003) derived the emission measure distribution of the upper transition region and corona of $\epsilon$ Eri. They modelled this distribution, and derived filling factors of the emitting material at the surface of the star from Fe IX to Fe XVI lines. They obtained filling factors that vary from $20 \%$ in the mid-transition region to $100 \%$ in the inner corona.

Houdebine \& Doyle (1994a,b) used $\mathrm{H}_{\alpha}$ and $\mathrm{H}_{\beta}$ to constrain the chromospheric structure of plages on AU Mic. They derived an upper limit of the plage filling factor of about 30\%. Their chromospheric model provides a lower limit to pressure because the quiescent region contribution was not taken into account. Houdebine (2009a, Paper X) confirmed the fact that this model does represent a lower limit in the case of another dM1e star, G1 867A. The quiescent region contribution was subtracted on this star, and the resulting $\mathrm{H}_{\alpha}$ plage profile shows no central self-reversal. According to the models of Houdebine \& Doyle (1994a), this result shows that the chromospheric pressure has been underestimated in the AU Mic model. The Balmer lines are of interest to derive a first guess to the plage filling factors on dMe stars because they have a high contrast between plages (strong emission) and quiescent regions (weak absorption or weak emission) (see Paper X).

Here, in the case of intermediate-activity dM1 stars, we can use none of the above constraints, either because we do not have the adequate observations (UV lines) or because the HeI and Balmer lines are not in emission. We therefore proceed in a different way.

\subsection{What is the contrast between quiescent and active regions?}

As we see below, the contrast between quiescent and active regions is important for constraining their chromospheric structures. In the solar case, this is about a factor of 5 in the lower chromospheric CaII and MgII resonance lines (e.g. Chapman 1980; Ayres et al. 1981). In the HeI lines, the contrast is higher, typically a factor of 16 (e.g., Smith et al. 2009) and may reach a factor of 30. This highlights the importance of HeI latter lines for determining the filling factors of active stars.

For $\mathrm{H}_{\alpha}$ and $\mathrm{H}_{\beta}$ in active dwarfs, the comparison between probable quiescent regions and active regions in Paper $\mathrm{X}$ shows that the contrast in these lines is high. If $\mathrm{H}_{\alpha}$ and $\mathrm{H}_{\beta}$ are slightly in emission in quiescent regions, the $\mathrm{H}_{\alpha}$ equivalent width in plages is about $9 \AA$. This infers a contrast factor for the $\mathrm{H}_{\alpha}$ line between quiescent and active regions of Gl 867A of about 10. This factor is even higher for $\mathrm{H}_{\beta}$ and the higher Balmer lines. This is significantly higher than the contrast in the $\mathrm{CaII}$ resonance lines in the solar case. The lack of the HeI $\lambda 5876$ line in emission for the spectra of relatively active dM1 stars (G1 205, Paper X; Gl 2, Gl 49, and G192-11A in this paper) indicates that there is a high contrast in this line, between quiescent and active regions on dM1e stars such as G1867A, similar to the Sun. This contrast factor may be greater than 10 .

A number of authors have attempted to constrain the properties of active regions on active stars (e.g., Walter et al. 1987; Neff et al. 1989; Pagano et al. 2001). These authors devised a technique, based on multi-Gaussian fits to the $\mathrm{Mg}$ II h \& $\mathrm{k}$ lines, called the Spectral Imaging Technique, to determine the extent and surface intensities of plages in RS CVn systems such as AR Lac. Walter et al. (1987) found that two discrete features are present on the surface of the K star in the AR Lac system. They constrained their filling factors and positions, and found that the Mg II k surface flux of these plages is about five times the mean $\mathrm{Mg}$ II k surface flux of the K star. In 1985 observations, Neff et al. (1989) found that, three plages are present at the surface of the K star of the AR Lac system. From the fluxes and filling factor they derived, we find that the Mg II k surface fluxes in these plages are 4.3,12, and 2.2 times the emission from quiescent regions, respectively. Pagano et al. (2001) also inferred a contrast factor between 0.8 and 5.1 for plages in the $\mathrm{Mg}$ II h \& $\mathrm{k}$ lines on AR Lac. Therefore, the observations of plages in active systems show that the picture in active stars is very similar to the Sun. Because the MgII lines and the CaII lines are formed in the same part of the chromosphere, these findings for the MgII lines most probably also apply to the CaII lines.

\section{Two-component model chromospheres}

Many investigators consider the spectra of chromospheric lines in dMe stars without taking into account the inhomogeneous nature of the chromosphere, which of course we know is wrong. We know that the atmosphere is inhomogeneous from the photosphere to the corona from the observed rotational modulation. Chromospheric emission therefore, probably arises, by analogy with the sun, both from the quiescent regions and plages. The problem of course is to determine the contribution from each component.

The Balmer lines and the CaII resonance lines provide good constraints for the chromosphere. They are formed in slightly overlapping temperature domains. However, their differential variation in M1 dwarfs is unique (Paper VI). The $\mathrm{H}_{\alpha}$ line is, indeed, present weakly in absorption in very low activity stars: the absorption then progressively increases to a maximum, and then it decreases again (the line "fills in") until it reaches the emission domain. The CaII lines behave differently, monotonically increasing with increasing chromospheric temperature gradient. This difference provides a unique tool for constraining the chromosphere. 
However, an uncertainty remains concerning the filling factors of quiescent and active regions. This uncertainty can be lifted if one simply considers a constraint on the ratio of the surface intensities in the CaII lines in quiescent regions and active regions. We assume here that in relatively less active dM1 stars, the solar constraint holds, i.e., there is a contrast of about a factor of 5 between quiescent and active regions in the CaII lines. Therefore, one can simply relate the quiescent region equivalent width $E W_{\mathrm{QR}}$ to the total observed equivalent width $E W_{\mathrm{Obs}}$ in the CaII lines:

$E W_{\mathrm{Obs} .}^{\mathrm{CaII}}=5 \times f \times E W_{\mathrm{QR}}^{\mathrm{CaII}}+(1-f) \times E W_{\mathrm{QR}}^{\mathrm{CaII}}$,

where $f$ is the plage filling factor. Howevrer, $f$ must also comply with the constraint from the $\mathrm{H}_{\alpha}$ line, i.e.

$E W_{\text {Obs. }}^{\mathrm{H}_{\alpha}}=f \times E W_{\text {Plage }}^{\mathrm{H}_{\alpha}}+(1-f) \times E W_{\mathrm{QR}}^{\mathrm{H}_{\alpha}}$,

where $E W_{\text {Plage }}^{\mathrm{H}_{\alpha}}$ and $E W_{\mathrm{QR}}^{\mathrm{H}_{\alpha}}$ are the $\mathrm{H}_{\alpha}$ equivalent widths of plages and quiescent regions respectively. These equivalent widths are related to the CaII line equivalent widths by models from Houdebine \& Stempels (1997). Therefore, for a given $\mathrm{H}_{\alpha}$ equivalent width in quiescent regions we derive the CaII equivalent widths for quiescent and active regions, and then derive the $\mathrm{H}_{\alpha}$ equivalent width for active regions. We then find that $E W_{\text {Plage }}^{\mathrm{H}_{\alpha}}$ is a function of $E W_{\mathrm{QR}}^{\mathrm{H}_{\alpha}}$

$E W_{\text {Plage }}^{\mathrm{H}_{\alpha}}=g\left(E W_{\mathrm{QR}}^{\mathrm{H}_{\alpha}}\right)$.

From Eq. (1) we derive the filling factor $f$;

$f=\frac{1}{4}\left(\frac{E W_{\mathrm{Obs} .}^{\mathrm{CaII}}}{E W_{\mathrm{QR}}^{\mathrm{CaII}}}-1\right)$.

However, since $E W_{\mathrm{QR}}^{\mathrm{CaII}}$ relates to $E W_{\mathrm{QR}}^{\mathrm{H}_{\alpha}}$ by means of models, we can write:

$E W_{\mathrm{QR}}^{\mathrm{CaII}}=h\left(E W_{\mathrm{QR}}^{\mathrm{H}_{\alpha}}\right)$.

From Eqs. (2), (3), and (5), one can then easily ascertain that $E W_{\text {Obs. }}^{\mathrm{H}_{\alpha}}$ is simply a function of $E W_{\mathrm{QR}}^{\mathrm{H}_{\alpha}}$

$E W_{\text {Obs. }}^{\mathrm{H}_{\alpha}}=\frac{1}{4}\left(\frac{E W_{\mathrm{Obs} .}^{\mathrm{CaII}}}{h\left(E W_{\mathrm{QR}}^{\mathrm{H}_{\alpha}}\right)}-1\right)\left(g\left(E W_{\mathrm{QR}}^{\mathrm{H}_{\alpha}}\right)-E W_{\mathrm{QR}}^{\mathrm{H}_{\alpha}}\right)+E W_{\mathrm{QR}}^{\mathrm{H}_{\alpha}}$.

We increase the $\mathrm{H}_{\alpha}$ equivalent width of quiescent regions until the observed $\mathrm{H}_{\alpha}$ equivalent width is matched. Therefore, the chromospheres of quiescent and active regions are described completely by our models in Paper VI when we add the additional constraint of a factor of 5 contrast between quiescent regions and plages in the CaII lines.

We calculated the function $g\left(E W_{\mathrm{QR}}^{\mathrm{H}_{\alpha}}\right)$ as a function of $E W_{\mathrm{QR}}^{\mathrm{H}_{\alpha}}$ for all the model chromospheres of Paper VI. The results are tabulated in Table 2.

Equation (6) produces diagrams of $E W_{\text {Obs. }}^{\mathrm{H}_{\alpha}}$ as a function of $E W_{\mathrm{QR}}^{\mathrm{H}_{\alpha}}$ as presented in Figs. 1, 3, 5 and 7. The intersections of these curves with the observed $\mathrm{H}_{\alpha}$ equivalent width provide the possible solutions. Because of the nature of the variation in the $\mathrm{H}_{\alpha}$ equivalent width as a function of activity level (Paper VI), we have often two solutions. One of these solutions may however be impossible. Our method gives exact solutions for the $\mathrm{CaII} \mathrm{H} \& \mathrm{~K}$ mean equivalent width and the $\mathrm{H}_{\alpha}$ equivalent width. However, although the equivalent widths are reproduced, some differences between the observed and theoretical profiles remain as we show below.

When we obtain a viable solution, we compute the CaII H $\& \mathrm{~K}$ mean equivalent width for quiescent regions from the tabulated values of Table 2 . When the solution in $E W_{\text {Obs. }}^{\mathrm{H}_{\alpha}}$ lies between two models $i$ and $i+1$, we compute the CaII H \& $\mathrm{K}$ mean equivalent width by giving weights to these models defined by

$$
\begin{aligned}
E W_{\mathrm{QR}}^{\mathrm{CaII}}= & \frac{E W_{\text {Obs. }}^{\mathrm{H}_{\alpha}}-E W_{i}^{\mathrm{H}_{\alpha}}}{E W_{i+1}^{\mathrm{H}_{\alpha}}-E W_{i}^{\mathrm{H}_{\alpha}}} \times E W_{i}^{\mathrm{CaII}} \\
& +\frac{E W_{\text {Obs. }}^{\mathrm{H}_{\alpha}}-E W_{i+1}^{\mathrm{H}_{\alpha}}}{E W_{i+1}^{\mathrm{H}_{\alpha}}-E W_{i}^{\mathrm{H}_{\alpha}}} \times E W_{i+1}^{\text {CaII }} .
\end{aligned}
$$

We compute the CaII $\mathrm{H} \& \mathrm{~K}$ mean equivalent width for active regions and the $\mathrm{H}_{\alpha}$ equivalent width for quiescent and active regions in the same way. To compute the theoretical profiles, we proceed in the same way; the profiles of models $i$ and $i+1$ are given by weights related to their equivalent widths. We multiply these profiles by their respective weights (in \%) and add the resulting profiles to obtain the theoretical profile. The profiles of quiescent and active regions are also given weights according to the active-region filling factor. The resulting profiles are shown for each star below in Figs. 2, 4, 6, 8, 10, 12, 14, and 16. These profiles are added to obtain the total theoretical profiles that are compared with the observed profiles.

One major constraint of this method is that it applies only, for the moment, to near-solar metallicity stars, because our model chromospheres in Paper VI were calculated only for solar metallicity. Among the target dM1 stars that we observed in Paper VI, we selected nine stars of near-solar metallicity. We now consider each star in detail and propose a two-component model chromosphere for each star.

We use the PRD model calculations of Houdebine \& Stempels (1997, Paper VI). Houdebine \& Stempels (1997) computed the CaII H \& K line profiles as well as the HI Balmer line profiles for both CRD and PRD, and for a grid of $30 \mathrm{dM} 1$ stellar model chromospheres from basal chromospheres to the most active dM1e chromospheres. There are important differences between CRD and PRD calculations both for the CaII lines and the Balmer lines. The PRD affects the Balmer lines because the HI Lyman lines and continuum irradiate the upper chromosphere where the Balmer lines are formed, i.e., the effect of PRD on the Lyman lines in turn affects the Balmer lines. The most important effects are on the highest optical-depth Balmer lines such as $\mathrm{H}_{\alpha}$ and $\mathrm{H}_{\beta}$ (see Paper VI and Houdebine \& Panagi 1990).

We also attempt to derive uncertainties in our model solutions for each star. These uncertainties are caused by uncertainties in both the measurements of the $\mathrm{H}_{\alpha}$ equivalent width and the CaII mean equivalent width. We consider either the $\pm \sigma$ domain or the $\pm 3 \sigma$ domain depending on the initial solution that we obtained. The uncertainties that we derive for the models emphasize the importance of simultaneous high $\mathrm{S} / \mathrm{N}$ ratio observations to this modelling. We show that it is possible to achieve a precision of about $\pm 0.1 \AA$ for the CaII line measure and about $\pm 0.015 \AA$ for the $\mathrm{H}_{\alpha}$ line measure (Table 1).

\section{Model chromosphere for GI 205}

We begin to analyze G1 205 to compare the results of the present method with the earlier results of Paper X. Gl 205 is slightly metal-rich $(+0.101 \mathrm{dex})$ and was not in the original selection of our solar metallicity M1 dwarfs. Nevertheless, its analysis 
E. R. Houdebine: Chromospheres of nine dM1 stars

Table 2. $\mathrm{H}_{\alpha}$, CaII H \& K equivalent widths and fluxes, and function values (see text) for the models of Paper VI.

\begin{tabular}{|c|c|c|c|c|c|c|c|}
\hline $\begin{array}{l}\text { Model } \\
\text { No }\end{array}$ & $\begin{array}{c}E W_{\mathrm{QR}}^{\mathrm{H}_{\alpha}} \\
(\AA)\end{array}$ & $\begin{array}{c}F_{\text {CaII H }} \\
\mathrm{erg} / \mathrm{s} / \mathrm{cm}^{2}\end{array}$ & $\begin{array}{c}E W_{\text {CaIl H }} \\
(\AA)\end{array}$ & $\begin{array}{c}F_{\text {CaII K }} \\
\mathrm{erg} / \mathrm{s} / \mathrm{cm}^{2}\end{array}$ & $\begin{array}{c}E W_{\text {CaII K }} \\
(\AA)\end{array}$ & $\begin{array}{c}\langle E W \text { CaII H \& K }\rangle \\
(\AA)\end{array}$ & $\begin{array}{c}g\left(E W_{\mathrm{QR}}^{\mathrm{H}_{\alpha}}\right) \\
(\AA)\end{array}$ \\
\hline III-1 & -25.4 & 3.51 & -43.9 & $4.46 \mathrm{e}-5$ & -55.75 & -49.83 & - \\
\hline III-2 & -13.5 & $2.352 \mathrm{e}-5$ & -29.4 & 3.037 & -37.96 & -33.68 & - \\
\hline III-3 & -6.71 & 1.522 & -19.0 & 1.924 & -24.05 & -21.53 & - \\
\hline III-4 & -2.52 & $6.751 \mathrm{e}-6$ & -8.44 & $9.513 e-6$ & -11.89 & -10.17 & -26.2 \\
\hline III-5 & -1.35 & 4.625 & -5.78 & 6.659 & -8.32 & -7.05 & -14.7 \\
\hline III-6 & -0.47 & 3.524 & -4.41 & 5.482 & -6.85 & -5.63 & -10.4 \\
\hline III-7 & 0.056 & 2.326 & -2.91 & 3.644 & -4.56 & -3.74 & -5.66 \\
\hline III-8 & 0.195 & 1.863 & -2.33 & 3.054 & -3.82 & -3.08 & -4.45 \\
\hline III-9 & 0.328 & 1.495 & -1.87 & 2.464 & -3.08 & -2.48 & -3.34 \\
\hline III-10 & 0.422 & 1.228 & -1.54 & 2.061 & -2.58 & -2.06 & -2.57 \\
\hline III-11 & 0.484 & $9.935 \mathrm{e}-7$ & -1.24 & 1.692 & -2.12 & -1.68 & -1.86 \\
\hline III-12 & 0.525 & 8.222 & -1.03 & 1.407 & -1.76 & -1.40 & -1.32 \\
\hline III-13 & 0.551 & 6.691 & -0.836 & 1.184 & -1.48 & -1.16 & -0.575 \\
\hline III-14 & 0.559 & 5.609 & -0.701 & $9.873 e-7$ & -1.23 & -0.967 & -0.252 \\
\hline III-15 & 0.572 & 4.655 & -0.582 & 8.170 & -1.02 & -0.801 & -0.021 \\
\hline III-16 & 0.576 & 3.871 & -0.484 & 6.990 & -0.874 & -0.679 & 0.129 \\
\hline III-17 & 0.573 & 3.194 & -0.399 & 5.827 & -0.728 & -0.564 & 0.253 \\
\hline III-18 & 0.560 & 2.725 & -0.341 & 4.904 & -0.613 & -0.477 & 0.352 \\
\hline III-19 & 0.553 & 2.291 & -0.286 & 4.245 & -0.531 & -0.409 & 0.425 \\
\hline III-20 & 0.533 & 1.938 & -0.242 & 3.567 & -0.446 & -0.344 & 0.478 \\
\hline III-21 & 0.516 & 1.625 & -0.203 & 2.952 & -0.369 & -0.286 & 0.520 \\
\hline III-22 & 0.478 & 1.162 & -0.145 & 2.085 & -0.261 & -0.203 & 0.557 \\
\hline III-23 & 0.440 & $8.738 \mathrm{e}-8$ & -0.109 & 1.513 & -0.189 & -0.149 & 0.573 \\
\hline III-24 & 0.390 & 6.487 & $-8.11 \mathrm{e}-2$ & 1.114 & -0.139 & -0.110 & 0.570 \\
\hline III-25 & 0.347 & 5.010 & $-6.26 e-2$ & $8.329 \mathrm{e}-8$ & -0.104 & -0.0833 & 0.554 \\
\hline III-26 & 0.300 & 4.692 & $-5.87 e-2$ & 8.309 & -0.103 & -0.0809 & 0.552 \\
\hline III-27 & 0.222 & 2.736 & $-3.42 e-2$ & 4.175 & $-5.23 e-2$ & -0.0433 & 0.484 \\
\hline III-28 & 0.134 & 2.145 & $-2.68 \mathrm{e}-2$ & 3.120 & $-3.90 \mathrm{e}-2$ & -0.0329 & 0.452 \\
\hline III-29 & 0.051 & 1.491 & $-1.86 \mathrm{e}-2$ & 1.987 & $-2.48 \mathrm{e}-2$ & -0.0217 & 0.388 \\
\hline III-30 & 0.005 & $7.714 \mathrm{e}-9$ & $-9.64 e-3$ & $9.985 \mathrm{e}-9$ & $-1.25 \mathrm{e}-2$ & -0.0111 & 0.247 \\
\hline
\end{tabular}

is informative because this star has almost the same radius as G1 867A which is a dM1e star and the two stars can be compared (Paper X). Here we assume a solar metallicity for G1 205 to use our models of Paper VI. This approximation should be reasonable.

The star G1 205 has a rather average CaII equivalent width $\left(-1.43 \AA\right.$, Paper VI) and a large $\mathrm{H}_{\alpha}$ equivalent width of $0.459 \AA$. We compute $E W_{\text {Obs. }}^{\mathrm{H}_{\alpha}}$ from Eq. (6) as a function of $E W_{\mathrm{QR}}^{\mathrm{H}_{\alpha}}$ for the CaII line equivalent width of $-1.43 \AA$ A. In Fig. 1, we show $E W_{\text {Obs. }}^{\mathrm{H}_{\alpha}}$ as a function of $E W_{\mathrm{QR}}^{\mathrm{H}_{\alpha}}$. The range of our measurements of the $\mathrm{H}_{\alpha}$ equivalent width for G1205 is also indicated. One can see that there are two solutions possible for Gl 205; a low solution (low CaII $E W$ ) and a high solution (high CaII $E W$ ), because the $E W_{\text {Obs. }}^{\mathrm{H}_{\alpha}}$ curve equals twice the observed $\mathrm{H}_{\alpha}$ equivalent width of $0.459 \AA$ (Fig. 1). However, the "low" solution infers a plage filling factor of greater than $100 \%$ and is therefore impossible for the assumptions that we use here. For different assumptions, notably for the contrast factor, we could obtain two solutions (see below for G1 49 and G 192-11A for instance). The "high" solution gives CaII mean equivalent widths of $-0.884 \AA$ and $-4.42 \AA$ for the quiescent regions and plage regions, respectively. The corresponding filling factor for plages is $15 \%$. The spectral line equivalent widths for quiescent and active regions are summarized in Table 4.

We show the CaII $\mathrm{H} \& \mathrm{~K}$, and $\mathrm{H}_{\alpha}$ profiles for the quiescent region contribution, the plage contribution, the total surface of the star, and the observations in Fig. 2. The total theoretical profiles were convolved with the ELODIE instrumental profile and a Gaussian of $F W H M 1.7 \mathrm{~km} \mathrm{~s}^{-1}(v \sin i)$. The fits to the spectral lines are relatively good for the $\mathrm{H}_{\alpha}$ and CaII $\mathrm{H}$ lines. In particular, our modelling approach implies that the CaII mean equivalent width is fitted perfectly. However, the theoretical CaII K profile is slightly smaller than the observed CaII $\mathrm{K}$ profile, because the theoretical $\mathrm{K}$ to $\mathrm{H}$ line ratio does not exactly match the observed ratio. This slight difference between models and observations was also noted in Paper VI. The theoretical profiles are more optically thick than the observed profiles. To date, we have no explanation for this difference.

In Fig. 2, we note that the wings of the $\mathrm{H}_{\alpha}$ profile are slightly stronger than the observed $\mathrm{H}_{\alpha}$ wings. As we see in Sect. 14, this is probably caused by blending with photospheric absorption lines. Unfortunately, at this stage we cannot remove these blends. As a result of stronger wings, our modelling of the equivalent width tries to compensate by increasing the absorption at the center of the line. Our model then yields an absorption at the line centre that is slightly too strong.

In Fig. 2, we can see that the $\mathrm{H}_{\alpha}$ profile is dominated by the contribution from quiescent regions. The $\mathrm{H}_{\alpha}$ profile from active regions is slightly in emission, but because of the relatively small filling factor, its contribution is less than from quiescent regions.

Our theoretical CaII H \& K profiles show a weak central absorption due to the large optical thickness of the chromosphere in these lines. Our observed CaII H profile also shows some weak central absorption, and the CaII K observed profile is rather flat at line center. Unfortunately, our CaII observations have a poor $\mathrm{S} / \mathrm{N}$ ratio and better observations are required. Nevertheless, higher $\mathrm{S} / \mathrm{N}$ ratio observations of the $\mathrm{CaII} \mathrm{H}$ line in $\mathrm{AX}$ Mic and Gl 884 (dK7 stars) show that there is indeed a central absorption 


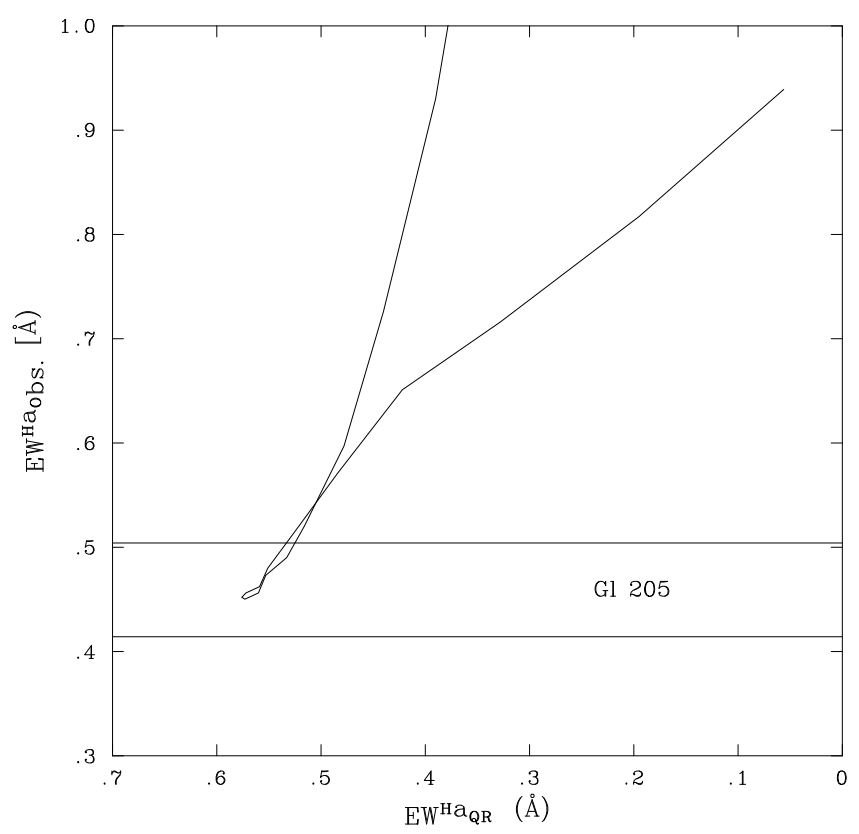

Fig. 1. We show $E W_{\mathrm{Obs}}^{\mathrm{H}_{\alpha}}$ as a function of $E W_{\mathrm{OR}}^{\mathrm{H}_{\alpha}}$ for Gl 205. The range of our measurements of the $\mathrm{H}_{\alpha}$ equivalent width for Gl 205 is also indicated as the two horyzontal lines that represent the $\pm 3 \sigma$ limit on the $\mathrm{H}_{\alpha}$ equivalent width measurement. One can see that there are two solutions possible for G1 205. However, the "lower" solution gives a plage filling factor greater than 1 and is therefore impossible.

(Paper VIII). Our CaII H \& K profiles of G1 205 are reproduced relatively well considering the noise in the spectra. We note that the main problem in these spectra is in determining the uncertainty due to the continuum level, and consequently the measurements of the equivalent widths of the CaII lines. Since our modelling depends on this mean equivalent width determination, this also affects our theoretical $\mathrm{H}_{\alpha}$ profile.

The contributions of the quiescent regions and the plages regions to the total CaII H \& K profiles are comparable (Fig. 2). This emphasizes the difference in the behaviour of these lines and of the $\mathrm{H}_{\alpha}$ line. The CaII lines are far more sensitive than the $\mathrm{H}_{\alpha}$ line to the contribution from the plages of intermediateactivity dM1 stars.

If one considers the uncertainty in the CaII $\mathrm{H} \& \mathrm{~K}$ equivalent widths, our fits to observations are good overall. There is also a large uncertainty on the $\mathrm{H}_{\alpha}$ equivalent width, because of blends and the uncertainty in the determination of the continuum (see Paper VI).

Our best model fit is comparable to that derived in Paper X. The present models for quiescent regions and plages have a slightly higher chromospheric pressure, but they are not very different. The main difference from the model of Paper $\mathrm{X}$ is related to the plage filling factor, which is presently about half. However, because the plage contribution to the $\mathrm{H}_{\alpha}$ profile is relatively small, the resulting global $\mathrm{H}_{\alpha}$ profile is not much different.

For the error domain of the CaII mean equivalent width, we consider the solution for the lower value of the CaII mean equivalent width of $-1.12 \AA$. We also find a solution for the maximum value of $-1.74 \AA$. From these two solutions, we estimate the uncertainties in our model. We find that the resulting uncertainties in the model are: $\delta \mathrm{H}_{\alpha}^{\mathrm{QR}}= \pm 0.003 \AA, \delta \mathrm{H}_{\alpha}^{\mathrm{Plage}}= \pm 0.024 \AA$, $\delta \mathrm{CaII}^{\mathrm{QR}}= \pm 0.021 \AA, \delta \mathrm{CaII}^{\text {Plage }}= \pm 0.09 \AA, \delta f / f= \pm 7 \%$, $\delta \log (M)_{\mathrm{QR}}= \pm 0.011$ and $\delta \log (M)_{\text {Plage }}= \pm 0.010$. These figures are rather small considering the large uncertainty in the CaII Mean equivalent width. We also list these values in Table 3.

As far as the uncertainty in $\mathrm{H}_{\alpha}$ is concerned, we consider our solution for the CaII mean equivalent width of $-1.43 \AA$. We then consider the $\pm \sigma$ interval in $\mathrm{H}_{\alpha}$ centered on $0.459 \AA$ (Fig. 1). We have a solution for the upper value of $\mathrm{H}_{\alpha}$ of $0.474 \AA$. For the lower value of $0.444 \AA$, we have no direct solution, but instead, as an estimate, we take the solution for an equivalent width of $0.450 \AA$, which is very close, and corresponds to the minimum of the curve in Fig. 1. From these two solutions, we estimate the uncertainties in our model due to $\mathrm{H}_{\alpha}$. We find that the resulting uncertainties in the model are $\delta \mathrm{H}_{\alpha}^{\mathrm{QR}}= \pm 0.01 \AA, \delta \mathrm{H}_{\alpha}^{\text {Plage }}= \pm 0.34 \AA$, $\delta \mathrm{CaII}^{\mathrm{QR}}= \pm 0.27 \AA, \delta \mathrm{CaII}^{\text {Plage }}= \pm 1.3 \AA, \delta f / f= \pm 15 \%$, $\delta \log (\mathrm{M})_{\mathrm{QR}}= \pm 0.086$ and $\delta \log (M)_{\text {Plage }}= \pm 0.084$. We also list these values in Table 3 . These values are not very large and correspond to one of the lowest total uncertainties for our studied stars (Table 3). In particular, the total uncertainty on the column mass is fairly small and restricts considerably the possible solutions for Gl 205. In the case of Gl 205 we can say that the model chromospheres are rather well determined.

\section{Model chromosphere for GI 2}

Houdebine \& Stempels (1997) provided a mean CaII line equivalent width of $-1.28 \AA$ for severall observations. In Paper VI, with our own observations, we found an equivalent width of $-1.57 \AA$, the value that we prefer because the $\mathrm{H}_{\alpha}$ and the CaII lines were observed simultaneously. This is very important as we see below.

We compute $E W_{\text {Obs. }}^{\mathrm{H}_{\alpha}}$ from Eq. (6) as a function of $E W_{\mathrm{QR}}^{\mathrm{H}_{\alpha}}$ for the two observed values of the CaII lines, $-1.28 \AA$ and $-1.57 \AA$. This allows us to estimate the effect of the CaII mean equivalent width on $E W_{\text {Obs. }}^{\mathrm{H}_{\alpha}}$. We show the resulting curves in Fig. 3. As we can see in this figure, the curves, and especially the minimum value of $E W_{\text {Obs. }}^{\mathrm{H}_{\alpha}}$, are very sensitive to the observed mean CaII equivalent width. For a CaII equivalent width of $-1.28 \AA$, we find no solution for G12. For a CaII equivalent width of $-1.57 \AA$, we also find no solution. The lowest value of the CaII mean equivalent width for which we have a solution is $-1.7 \AA$. This falls within the measurement errors. Therefore, we have one solution that corresponds to the minimum of the curve. The minimum predicted $E W_{\text {Obs. }}^{\mathrm{H}_{\alpha}}$ is $0.405 \AA$, which is not very far from the $0.353 \AA$ observed. For this model, the theoretical CaII mean equivalent width is $-0.967 \AA$ and $-4.84 \AA$ for quiescent regions and active regions, respectively. We obtain a plage filling factor of $19 \%$ for these values, which is comparable to the value determined for Gl205, a star with a similar activity level.

We show in Fig. 4 the theoretical and observed profiles. The total theoretical profiles were convolved with the ELODIE instrumental profile and a Gaussian of FWHM $1.2 \mathrm{~km} \mathrm{~s}^{-1}(v \sin i)$. In the case of $\mathrm{H}_{\alpha}$, the fit is reasonable except at the line center where the theoretical model shows a stronger absorption than the observed profile. For our model, the entire profile is dominated by quiescent regions, as in G1 205.

For the CaII lines, our model underestimates the CaII H flux and overestimates the CaII $\mathrm{K}$ flux. This is again due to some difference in the line ratio between models and observations. We do not see a central self-reversal in the CaII line observations. However, higher $\mathrm{S} / \mathrm{N}$ ratio observations are necessary to reach any conclusion. In the case of G12, the CaII line profiles are largely dominated by the contribution from active regions. 
E. R. Houdebine: Chromospheres of nine dM1 stars
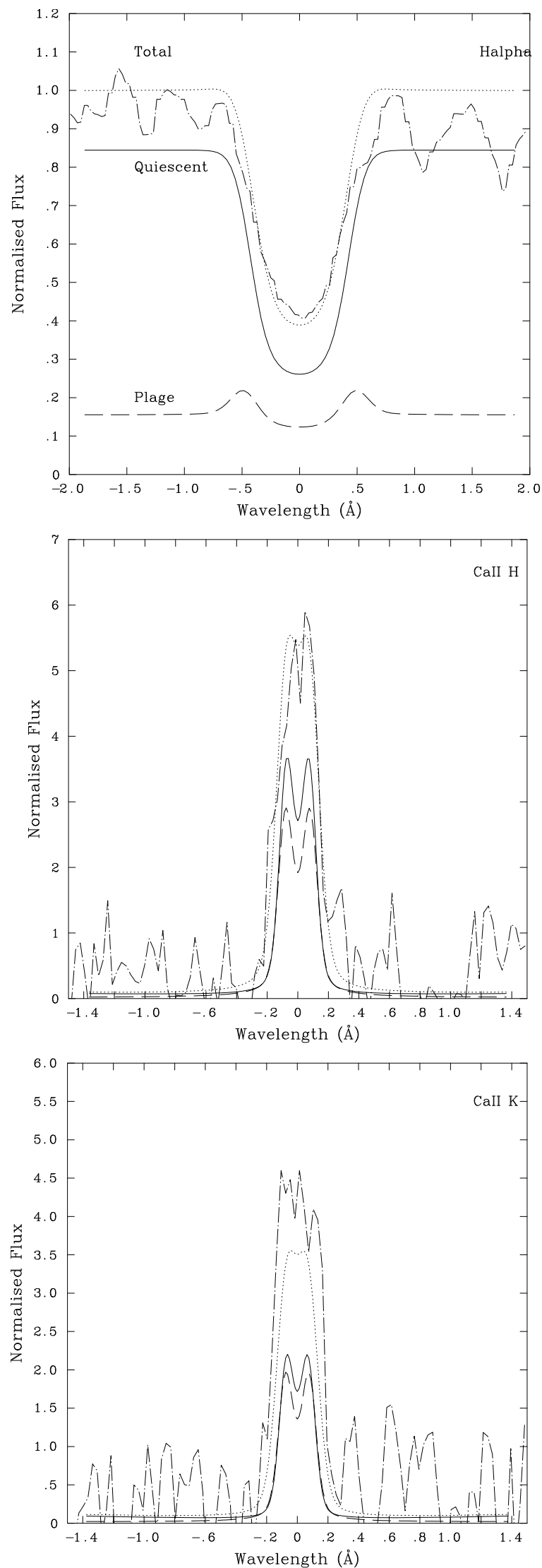

Fig. 2. In this figure we show our best fits to the $\mathrm{H}_{\alpha}$, CaII H and CaII $\mathrm{K}$ lines for Gl205. The quiescent region contribution is shown as a continuous line, the plage contribution as a dashed line, the total theoretical profiles as a dotted line, and the observed profiles as a dashed-dotted line.

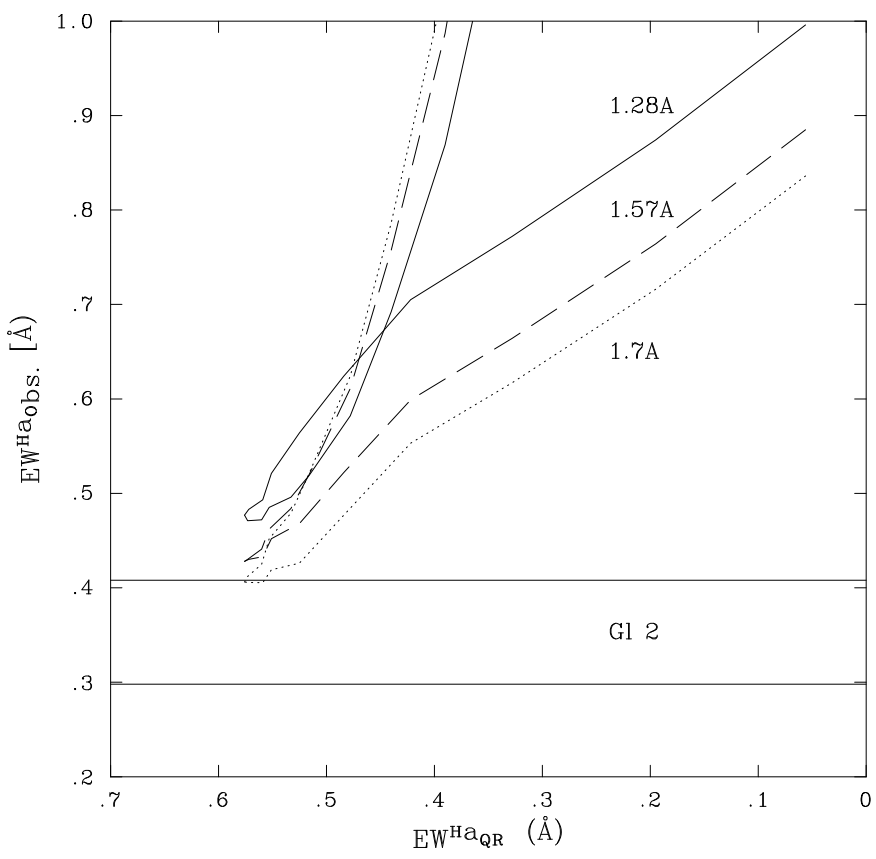

Fig. 3. We show $E W_{\mathrm{Obs} \text {. }}^{\mathrm{H}_{\alpha}}$ as a function of $E W_{\mathrm{QR}}^{\mathrm{H}_{\alpha}}$ for Gl 2 and for three values of the mean CaII $\mathrm{H} \& \mathrm{~K}$ equivalent widths: $-1.28 \AA,-1.57 \AA$ and $-1.7 \AA$. The range of our measurements of the $\mathrm{H}_{\alpha}$ equivalent width for Gl 2 is also indicated as the two horyzontal lines that represent the $\pm 3 \sigma$ limit on the $\mathrm{H}_{\alpha}$ equivalent width measurement. One can see that there is only one solution possible for Gl 2 for the CaII equivalent width of $-1.7 \AA$.

The CaII line equivalent widths are of the same order for G1 2 and $\mathrm{Gl} 205$. However, their $\mathrm{H}_{\alpha}$ profiles differ significantly bacause in Gl 205 the quiescent regions and plages have a slightly higher pressure. The plages are also slightly more extended on the surface of Gl 2 than on Gl 205. Nevertheless, both stars have comparable activity levels, and they are both intermediate activity stars with filled-in $\mathrm{H}_{\alpha}$ profiles. These differences between Gl 2 and Gl 205 emphasize the importance of two-component modelling to interpreting their spectra.

For the error domain of the CaII mean equivalent width, we have a solution for the upper value of the CaII mean equivalent width of $-1.84 \AA$. We use this solution to estimate the relevant uncertainties in our model. We find that the resulting uncertainties in the model are: $\delta \mathrm{H}_{\alpha}^{\mathrm{QR}}= \pm 0 \AA, \delta \mathrm{H}_{\alpha}^{\mathrm{Plage}}= \pm 0 \AA, \delta \mathrm{CaII}{ }^{\mathrm{QR}}=$ $\pm 0 \AA, \delta \mathrm{CaII}^{\text {Plage }}= \pm 0 \AA, \delta f / f= \pm 4 \%, \delta \log (M)_{\mathrm{QR}}= \pm 0$ and $\delta \log (M)_{\text {Plage }}= \pm 0$. These figures are null for many parameters because the two solutions are for the same column mass.

For the uncertainty in $\mathrm{H}_{\alpha}$, the problem is that we have no solution for the observed CaII mean equivalent width of $-1.57 \AA$ and there is almost no uncertainty in our solution at $-1.7 \AA$ due to $\mathrm{H}_{\alpha}$ because this solution is right at the edge of the $3 \sigma$ domain. Therefore, we cannot derive an uncertainty in our model for $\mathrm{H}_{\alpha}$.

\section{Model chromosphere for GJ 1010A}

GJ 1010A is a rather low activity M1 dwarf with a CaII H \& K mean equivalent width of $-0.61 \AA$. Because of the low $\mathrm{S} / \mathrm{N}$ ratio of our observations, the uncertainty in this measurement is large, mostly because of the uncertainty in the adjacent continuum. The mean equivalent width can be any value between $-0.12 \AA$ and $-1.1 \AA$ (Paper VI). The $\mathrm{H}_{\alpha}$ equivalent width for GJ 1010A is the lowest of all our near-solar metallicity M1 dwarfs. This 

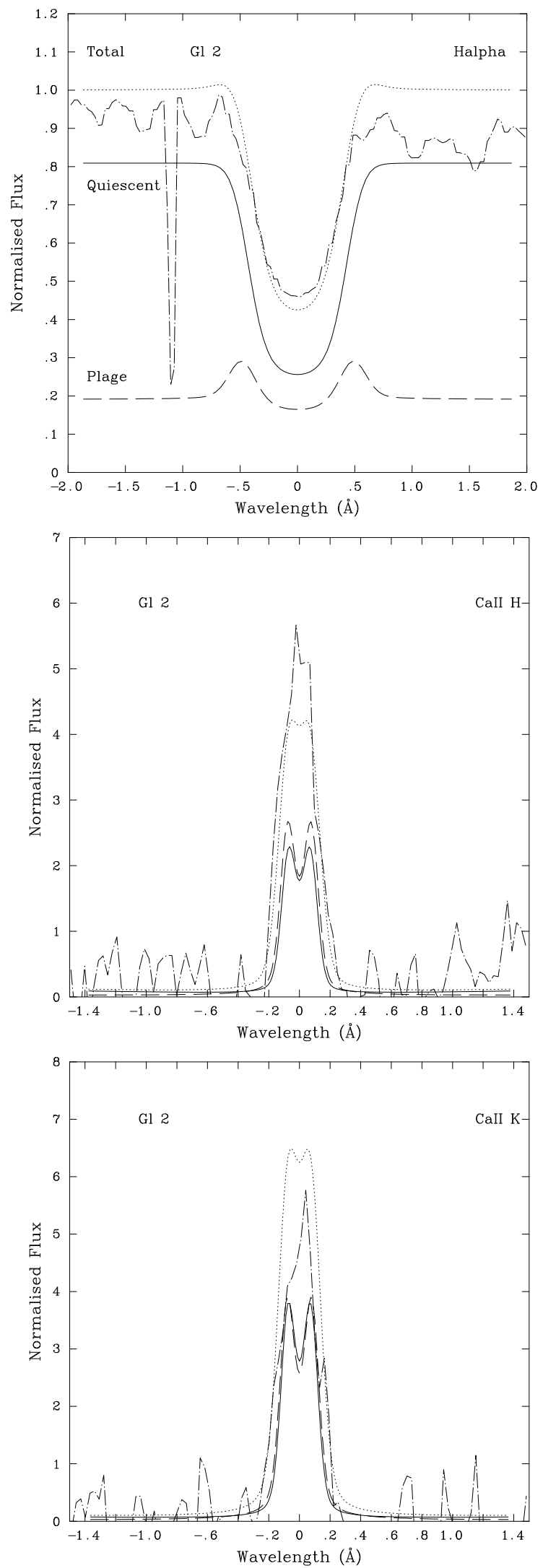

Fig. 4. In this figure we show our best fits to the $\mathrm{H}_{\alpha}$, CaII H and CaII K lines for Gl2. The quiescent region contribution is shown as a continuous line, the plage contribution as a dashed line, the total theoretical profiles as a dotted line, and the observed profiles as a dashed-dotted line.

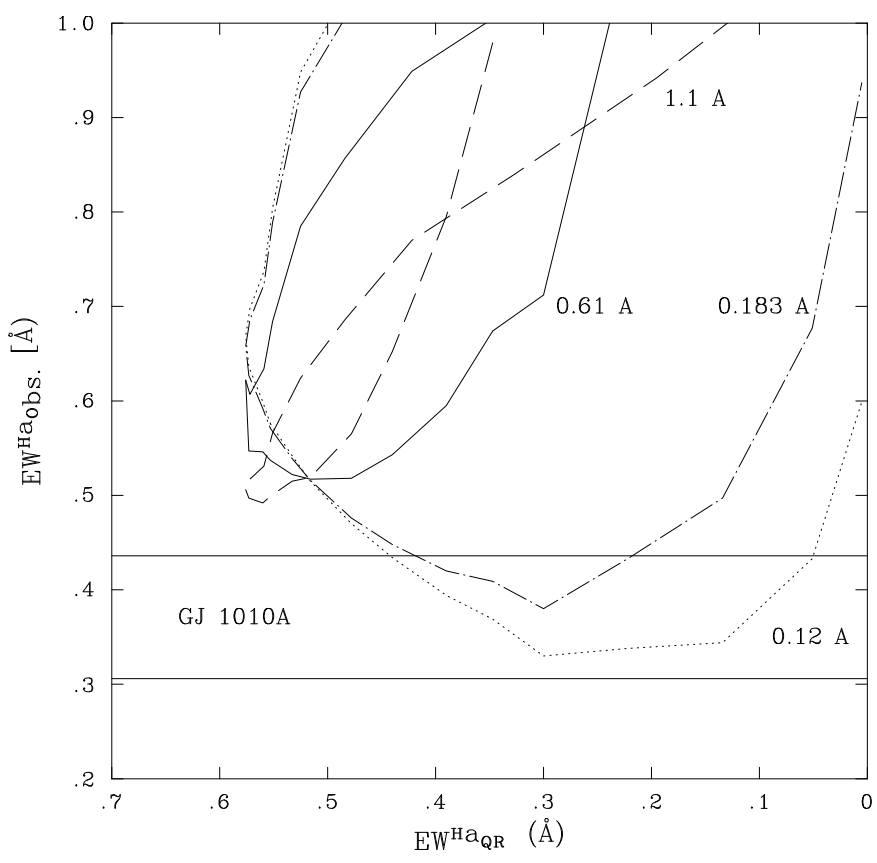

Fig. 5. We show $E W_{\mathrm{Obs}}^{\mathrm{H}_{\alpha}}$ as a function of $E W_{\mathrm{OR}}^{\mathrm{H}_{\alpha}}$ for GJ 1010A for severall values of the CaII mean equivalent width. The range of our measurements of the $\mathrm{H}_{\alpha}$ equivalent width for GJ 1010A is also indicated as the two horyzontal lines that represent the $\pm 3 \sigma$ limit on the $\mathrm{H}_{\alpha}$ equivalent width measurement. One can see that the best solution occurs for a CaII mean equivalent width of about $-0.18 \AA$ A.

low equivalent width further supports the case for a low activity star. Since this equivalent width is lower than that of GL 526, which has a lower mean CaII equivalent width, we may have over-estimated the CaII equivalent width for GJ 1010A.

We compute $E W_{\text {Obs. }}^{\mathrm{H}_{\alpha}}$ from Eq. (6) as a function of $E W_{\mathrm{QR}}^{\mathrm{H}_{\alpha}}$ for three values of the CaII line mean equivalent width, i.e., $-1.1 \AA,-0.61 \AA$, and $-0.12 \AA$. This allows us to estimate, in particular, the effect of the CaII mean equivalent width on $E W_{\text {Obs. }}^{\mathrm{H}_{\alpha}}$. We show the resulting curves in Fig. 5. As we can see in this figure, the curves, and especially the minimum value of $E W_{\text {Obs. }}^{\mathrm{H}_{\alpha}}$ are very sensitive to the observed mean CaII equivalent width. For a CaII equivalent width of $-0.61 \AA$, we find no solution for GJ 1010A. For a CaII equivalent width of $-1.1 \AA$, we find that the minimum of the curve is closer to the observed value, but we still have no solution for this upper value. For a CaII equivalent width of $-0.12 \AA$, we have two possible solutions. This suggests that our measurement of the CaII mean equivalent width has been overestimated. As the most likely solution, we choose the highest value of the CaII mean equivalent width for which we obtain a reasonable solution in Fig. 5. This value is about $-0.183 \AA$. We also show this curve in Fig. 5. The minimum predicted $E W_{\text {Obs. }}^{\mathrm{H}_{\alpha}}$ for this latter value of $-0.183 \AA$ of the CaII mean equivalent width is $0.380 \AA$, which is close to the observed value of $0.371 \AA$. The corresponding plage filling factor is $32 \%$.

We show the $\mathrm{CaII} \mathrm{H} \& \mathrm{~K}$, and $\mathrm{H}_{\alpha}$ profiles for the quiescent region contribution, the plage contribution, the total surface of the star, and the observations in Fig. 6. The total theoretical profiles were convolved with the ELODIE instrumental profile. The fits to the spectral lines are relatively good. We find that the theoretical CaII $\mathrm{H}$ profile is slightly larger than the observed $\mathrm{CaII} \mathrm{H}$ profile, and the theoretical CaII $\mathrm{K}$ profile is slightly 

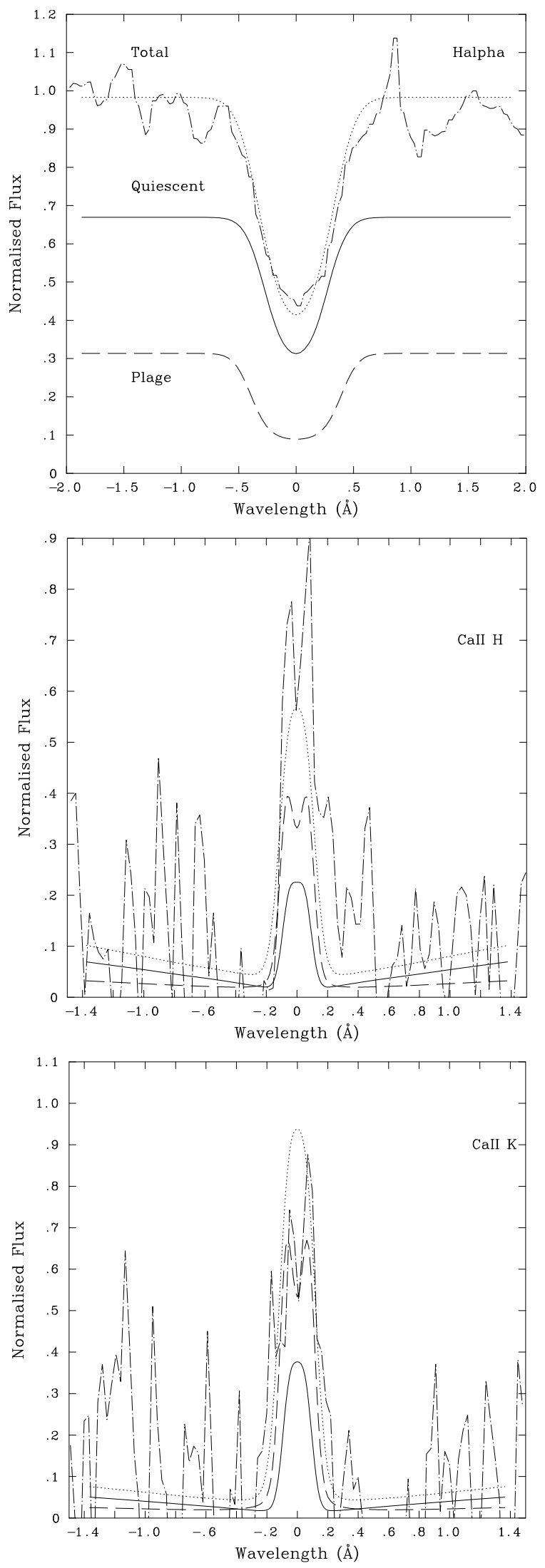

Fig. 6. In this figure, we show our best fits to the $\mathrm{H}_{\alpha}, \mathrm{CaIIH}$ and CaII K lines for GJ 1010A. The quiescent region contribution is shown as a continuous line, the plage contribution as a dashed line, the total theoretical profiles as a dotted line, and the observed profiles as a dashed-dotted line. smaller than the observed CaII $\mathrm{K}$ profile. This is because the theoretical $\mathrm{K}$ to $\mathrm{H}$ line ratio does not exactly match the observed ratio. However, the fit is reasonable if one considers the uncertainties in the equivalent width determinations and the noise in the spectra.

In Fig. 6, we note that the wings of the $\mathrm{H}_{\alpha}$ profile are more accurately fitted than for some of the other stars studied here. We note that we can reproduce an important feature of this low activity star; namely the $F W H M$ of the $\mathrm{H}_{\alpha}$ profile. Indeed, it was noted in Paper VI that the FWHM of low activity stars was significantly smaller than those of intermediate activity stars. Here our two-component model can reproduce rather well the whole $\mathrm{H}_{\alpha}$ profile.

In Fig. 6, we can see that the $\mathrm{H}_{\alpha}$ profile is dominated by the contribution from quiescent regions. The $\mathrm{H}_{\alpha}$ profile from active regions is strongly in absorption, but because of the relatively small filling factor, its contribution is smaller than from quiescent regions. We note the important differences in the shapes of the $\mathrm{H}_{\alpha}$ profiles of the quiescent regions and the active regions. The quiescent region profile is much narrower.

Our theoretical CaII H \& K profiles show no central absorption. We note that this central absorption in the theoretical profiles is much weaker than in the higher activity stars. This is because the chromosphere becomes more optically thin when the level of activity decreases (Paper VI). One can indeed see that the quiescent region CaII line profiles do not show any central absorption feature, when the active regions show a weak central absorption. The observed profiles may show a weak central absorption, in disagreement with our model, but this needs to be confirmed with higher $\mathrm{S} / \mathrm{N}$ ratio observations.

The contribution of the plage regions to the total CaII H \& K profiles is larger than the contribution of the quiescent regions (Fig. 6). It is interesting to note that the CaII line profiles are dominated by the contribution of plage regions throughout a large range of activity levels (see below). This is a direct consequence of the relatively large plage filling factors (typically $20 \%-40 \%$ ) that we find for all these stars.

For the error domain of the CaII mean equivalent width, we consider the solution for the lower value of the CaII mean equivalent width of $-0.12 \AA$. We also find a solution for the maximum value of $-0.22 \AA$. From these two solutions, we estimate the uncertainties in our model. We find that the resulting uncertainties in the model are $\delta \mathrm{H}_{\alpha}^{\mathrm{QR}}= \pm 0.14 \AA, \delta \mathrm{H}_{\alpha}^{\text {Plage }}= \pm 0.07 \AA$, $\delta \mathrm{CaII}{ }^{\mathrm{QR}}= \pm 0.04 \AA, \delta \mathrm{CaII}^{\mathrm{Plage}}= \pm 0.12 \AA, \delta f / f= \pm 26 \%$, $\delta \log (M)_{\mathrm{QR}}= \pm 0.40$, and $\delta \log (M)_{\text {Plage }}= \pm 0.25$. We also list these values in Table 3 .

As far as the uncertainty in $\mathrm{H}_{\alpha}$ is concerned, we consider our solution for the CaII mean equivalent width of $-0.183 \AA$. We then consider the $3 \sigma$ domain in $\mathrm{H}_{\alpha}$ (Fig. 5). We have a lower and an upper solution for $\mathrm{H}_{\alpha}=0.436 \AA$ (the upper limit of the $3 \sigma$ domain). From these two solutions, we estimate the uncertainties in our model related to $\mathrm{H}_{\alpha}$. We find that the resulting uncertainties in the model are $\delta \mathrm{H}_{\alpha}^{\mathrm{QR}}= \pm 0.10 \AA, \delta \mathrm{H}_{\alpha}^{\text {Plage }}= \pm 0.08 \AA$, $\delta \mathrm{CaII}^{\mathrm{QR}}= \pm 0.045 \AA, \delta \mathrm{CaII}^{\text {Plage }}= \pm 0.27 \AA, \delta f / f= \pm 36 \%$, $\delta \log (M)_{\mathrm{QR}}= \pm 0.28$, and $\delta \log (M)_{\text {Plage }}= \pm 0.25$. We also list these values in Table 3 .

\section{Model chromosphere for GI 49}

Gl 49 has a CaII mean equivalent width significantly larger than Gl 205 and Gl 2 (Table 1). Its $\mathrm{H}_{\alpha}$ equivalent width is rather small of only $0.254 \AA$. These equivalent widths indicate that this is another intermediate activity star with a filled-in $\mathrm{H}_{\alpha}$ profile. 
Table 3. Errors in the models due to the error measurements in the $\mathrm{H}_{\alpha}$ equivalent width and the CaII mean equivalent width (see text for each star).

\begin{tabular}{|c|c|c|c|c|c|c|c|}
\hline Star & $\begin{array}{c}\delta \text { Filling } \\
\text { Factor } \\
\sigma(\%) \\
\end{array}$ & $\begin{array}{c}\delta E W_{\mathrm{H}_{\alpha}} \\
\mathrm{QR} \\
\sigma(\AA) \\
\end{array}$ & $\begin{array}{c}\delta E W_{\mathrm{H}_{\alpha}} \\
\text { Plage } \\
\sigma(\AA) \\
\end{array}$ & $\begin{array}{c}\delta E W_{\text {CaII }} \\
\mathrm{QR} \\
\sigma(\AA) \\
\end{array}$ & $\begin{array}{c}\delta E W_{\text {CaII }} \\
\text { Plage } \\
\sigma(\AA) \\
\end{array}$ & $\begin{array}{c}\log (M) \\
\mathrm{QR} \\
\left(\mathrm{g} \mathrm{cm}^{-2}\right) \\
\end{array}$ & $\begin{array}{c}\delta \log (M) \\
\text { Plage } \\
\left(\mathrm{g} \mathrm{cm}^{-2}\right) \\
\end{array}$ \\
\hline $\begin{array}{c}\text { G12 } \\
\text { From CaII }( \pm 3 \sigma) \\
\text { GJ 1010A }\end{array}$ & 4 & 0 & 0 & 0 & 0 & 0 & 0 \\
\hline From CaII $( \pm 3 \sigma)$ & 26 & 0.14 & 0.07 & 0.04 & 0.12 & 0.40 & 0.25 \\
\hline From $\mathrm{H}_{\alpha}( \pm 3 \sigma)$ & 36 & 0.10 & 0.08 & 0.04 & 0.27 & 0.28 & 0.25 \\
\hline $\begin{array}{c}\text { Total } \\
\text { G1 } 205\end{array}$ & 62 & 0.24 & 0.15 & 0.08 & 0.39 & 0.68 & 0.50 \\
\hline From CaII $( \pm \sigma)$ & 7 & 0.003 & 0.024 & 0.02 & 0.09 & 0.011 & 0.010 \\
\hline From $\mathrm{H}_{\alpha}( \pm \sigma)$ & 15 & 0.01 & 0.34 & 0.27 & 1.3 & 0.086 & 0.084 \\
\hline $\begin{array}{l}\text { Total } \\
\text { G1 } 49\end{array}$ & 22 & 0.01 & 0.36 & 0.29 & 1.4 & 0.097 & 0.094 \\
\hline From CaII $( \pm \sigma)$ & 17 & 0.018 & 0.47 & 0.24 & 1.2 & 0.064 & 0.071 \\
\hline From $\mathrm{H}_{\alpha}( \pm \sigma)$ & 12 & 0.017 & 0.36 & 0.23 & 1.1 & 0.056 & 0.069 \\
\hline $\begin{array}{c}\text { Total } \\
\text { Gl 150.1B }\end{array}$ & 29 & 0.035 & 0.83 & 0.47 & 2.3 & 0.12 & 0.14 \\
\hline $\begin{array}{l}\text { From } \mathrm{H}_{\alpha}( \pm \sigma) \\
\quad \text { G1 } 229\end{array}$ & 40 & 0.007 & 0.19 & 0.17 & 0.9 & 0.082 & 0.067 \\
\hline From CaII $( \pm \sigma)$ & 0.5 & 0.007 & 0.048 & 0.044 & 0.22 & 0.025 & 0.019 \\
\hline From $\mathrm{H}_{\alpha}( \pm \sigma)$ & 41 & 0.026 & 0.15 & 0.15 & 0.75 & 0.096 & 0.085 \\
\hline $\begin{array}{c}\text { Total } \\
\text { G1 } 526\end{array}$ & 42 & 0.033 & 0.20 & 0.19 & 0.97 & 0.11 & 0.10 \\
\hline From CaII $( \pm \sigma)$ & 3.5 & 0.019 & 0.008 & 0.027 & 0.14 & 0.050 & 0.044 \\
\hline From $\mathrm{H}_{\alpha}( \pm 3 \sigma)$ & 43 & 0.037 & 0.037 & 0.078 & 0.39 & 0.099 & 0.088 \\
\hline $\begin{array}{c}\text { Total } \\
\text { G 192-11A }\end{array}$ & 46 & 0.056 & 0.045 & 0.11 & 0.53 & 0.14 & 0.13 \\
\hline From CaII $( \pm \sigma)$ & 18 & 0.011 & 0.39 & 0.19 & 0.97 & 0.056 & 0.052 \\
\hline From $\mathrm{H}_{\alpha}( \pm \sigma)$ & 7 & 0.008 & 0.19 & 0.13 & 0.64 & 0.031 & 0.036 \\
\hline $\begin{array}{c}\text { Total } \\
\text { Gl } 880\end{array}$ & 25 & 0.019 & 0.58 & 0.32 & 1.6 & 0.087 & 0.088 \\
\hline From CaII $( \pm \sigma)$ & 18 & 0.009 & 0.024 & 0.030 & 0.15 & 0.023 & 0.019 \\
\hline From $\mathrm{H}_{\alpha}( \pm \sigma)$ & 9 & 0.009 & 0.094 & 0.085 & 0.43 & 0.050 & 0.040 \\
\hline Total & 27 & 0.018 & 0.11 & 0.11 & 0.58 & 0.073 & 0.059 \\
\hline
\end{tabular}

We show $E W_{\text {Obs. }}^{\mathrm{H}_{\alpha}}$ from Eq. (6) as a function of $E W_{\mathrm{QR}}^{\mathrm{H}_{\alpha}}$ for the observed value of the CaII mean equivalent width in Fig. 7. One can see the important difference between this curve and the other curves inferred for Gl 205 and Gl 2, due to the rather large CaII mean equivalent width. Here, the lower and higher parts of the curve are well separated and the minimum is significantly below those of Gl 205 and Gl 2. For Gl 49, the minimum $E W_{\text {Obs. }}^{\mathrm{H}_{\alpha}}$ is only $0.202 \AA$. We find two solutions for Gl 49: the lower solution gives a plage filling factor of $26 \%$, and the higher solution gives a plage filling factor of only $9 \%$. Their respective CaII mean equivalent width for quiescent regions are $-1.17 \AA$ and $-1.76 \AA$. This latter value seems a bit high for quiescent regions, especially compared to those of G1 205 and G12. The filling factor derived for the lower solution is also more consistent with the values derived for G1 205 and G12. For these reasons we prefer the lower solution. In any case, the error domain in this model (29\%, Table 3 and below) includes the lower solution. These two solutions can therefore be considered as an estimate of the error in the models.

In Fig. 8, we show the theoretical profiles for the two solutions and the observed profiles. The total theoretical profiles were convolved with the ELODIE instrumental profile and a Gaussian of $F W H M 1.4 \mathrm{~km} \mathrm{~s}^{-1}(v \sin i)$. One can see that there is very little difference in the $\mathrm{H}_{\alpha}$ profiles for the two solutions. In the case of $\mathrm{H}_{\alpha}$, the fit is reasonable, except at the line center where the theoretical model shows stronger absorption than the observed profile. In the theoretical profiles, we can also note weak emission wings. These weak emission wings were already noted in the case of Gl 49, in the difference profile Gl 49-Gl 289 (Gl 289 is a low activity star) (Paper VI). Therefore, again, as in the case of Gl 205, if we do not detect these weak emission wings in the observed profile, this is because of probable blends in the wings of $\mathrm{H}_{\alpha}$. To derive a more robust fit, one would have to calculate the effects of these line blanketings on the $\mathrm{H}_{\alpha}$ profile, or attempt to subtract the contributions from these absorption lines by observing a basal profile of $\mathrm{H}_{\alpha}$.

We can see in Fig. 8 that the main contribution to the CaII lines comes from the active regions, as in G12. In this figure, we show only the lower solution for the CaII profiles. Our model underestimates the CaII H flux, as in G12. Nevertheless, our model yields reasonable results compared to observations. The observed profiles are flattened at the line center as in the models.

For the error domain of the CaII mean equivalent width, we consider the solution for the lower value of the CaII mean equivalent width of $-2.25 \AA$. We also find a solution for the maximum value of $-2.51 \AA$. From these two solutions, we estimate the uncertainties in our model. We find that the resulting uncertainties in the model are $\delta \mathrm{H}_{\alpha}^{\mathrm{QR}}= \pm 0.018 \AA, \delta \mathrm{H}_{\alpha}^{\text {Plage }}= \pm 0.47 \AA$,

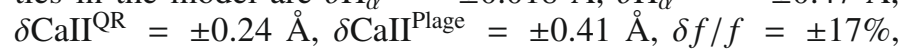
$\delta \log (M)_{\mathrm{QR}}= \pm 0.0641$ and $\delta \log (M)_{\text {Plage }}= \pm 0.071$. These values are typical if one considers the large uncertainty in the CaII Mean equivalent width. We also list these values in Table 3. 


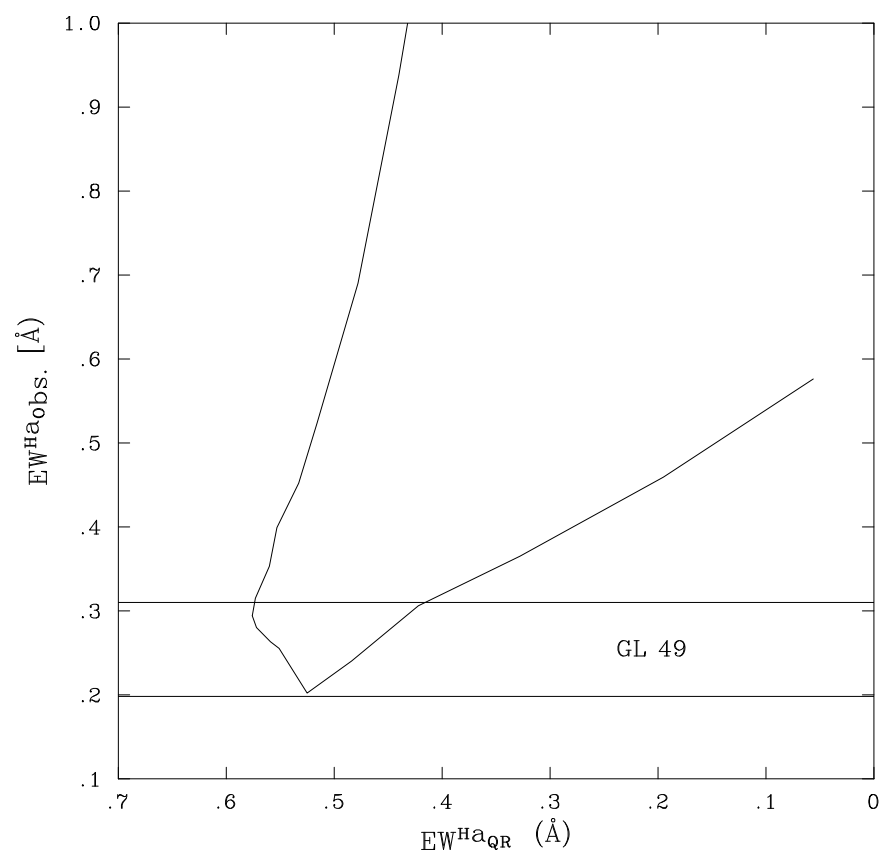

Fig. 7. We show $E W_{\mathrm{Obs}}^{\mathrm{H}_{\alpha}}$ as a function of $E W_{\mathrm{QR}}^{\mathrm{H}_{\alpha}}$ for $\mathrm{Gl} 49$ for the mean CaII H \& K equivalent width of $-2.38 \AA$. The range of our measurements of the $\mathrm{H}_{\alpha}$ equivalent width for Gl 49 is also indicated as the two horyzontal lines that represent the $\pm 3 \sigma$ limit on the $\mathrm{H}_{\alpha}$ equivalent width measurement. One can see that two solutions are possible.

As far as the uncertainty in $\mathrm{H}_{\alpha}$ is concerned, we consider our solution for the CaII mean equivalent width of $-1.43 \AA$. We then consider the $\pm \sigma$ domain in $\mathrm{H}_{\alpha}$. We have a solution for the upper limit of $\mathrm{H}_{\alpha}$ of $0.273 \AA$ and the lower limit of $0.235 \AA$. This is one of the rare stars in our sample for which we find solutions for the lower and upper values of both $\mathrm{H}_{\alpha}$ and CaII lines. From these two solutions, we estimate the uncertainties in our model related to $\mathrm{H}_{\alpha}$. We find that the resulting uncertainties in the model are: $\delta \mathrm{H}_{\alpha}^{\mathrm{QR}}= \pm 0.017 \AA, \delta \mathrm{H}_{\alpha}^{\text {Plage }}= \pm 0.36 \AA, \delta \mathrm{CaII}^{\mathrm{QR}}= \pm 0.23 \AA$, $\delta \mathrm{CaII}^{\text {Plage }}= \pm 1.2 \AA, \delta f / f= \pm 12 \%, \delta \log (M)_{\mathrm{QR}}= \pm 0.056$ and $\delta \log (M)_{\text {Plage }}= \pm 0.069$. These values are of the same order as those found for G1 205, and yield a total uncertainty that is one of the lowest of our stars (Table 3). In particular, the total uncertainty in the column mass is fairly small and therefore restricts considerably the possible solutions for Gl 49.

\section{Model chromosphere for GI 150.1B}

In Paper VI, we found an equivalent width of $-1.4 \AA$ for the mean CaII equivalent width of Gl 150.1B. The $\mathrm{H}_{\alpha}$ equivalent width of $0.439 \AA$ (Table 1) is also large for this star. This indicates that this star is of intermediate activity.

We compute $E W_{\text {Obs. }}^{\mathrm{H}_{\alpha}}$ from Eq. (6) as a function of $E W_{\mathrm{QR}}^{\mathrm{H}_{\alpha}}$ for the mean observed value of the CaII lines, and show the resulting curve in Fig. 9. We found one solution for this star, which corresponds to the minimum of the curve in Fig. 9. The minimum predicted $E W_{\text {Obs. }}^{\mathrm{H}_{\alpha}}$ is $0.454 \AA$, which is close to the $0.439 \AA$ value observed.

The theoretical CaII mean equivalent width for this model is $-0.564 \AA$ and $-2.82 \AA$ for quiescent regions and active regions, respectively. We obtain a plage filling factor of $37 \%$ for these values, which is one of the largest filling factors that we have found for $\mathrm{dM} 1$ stars.
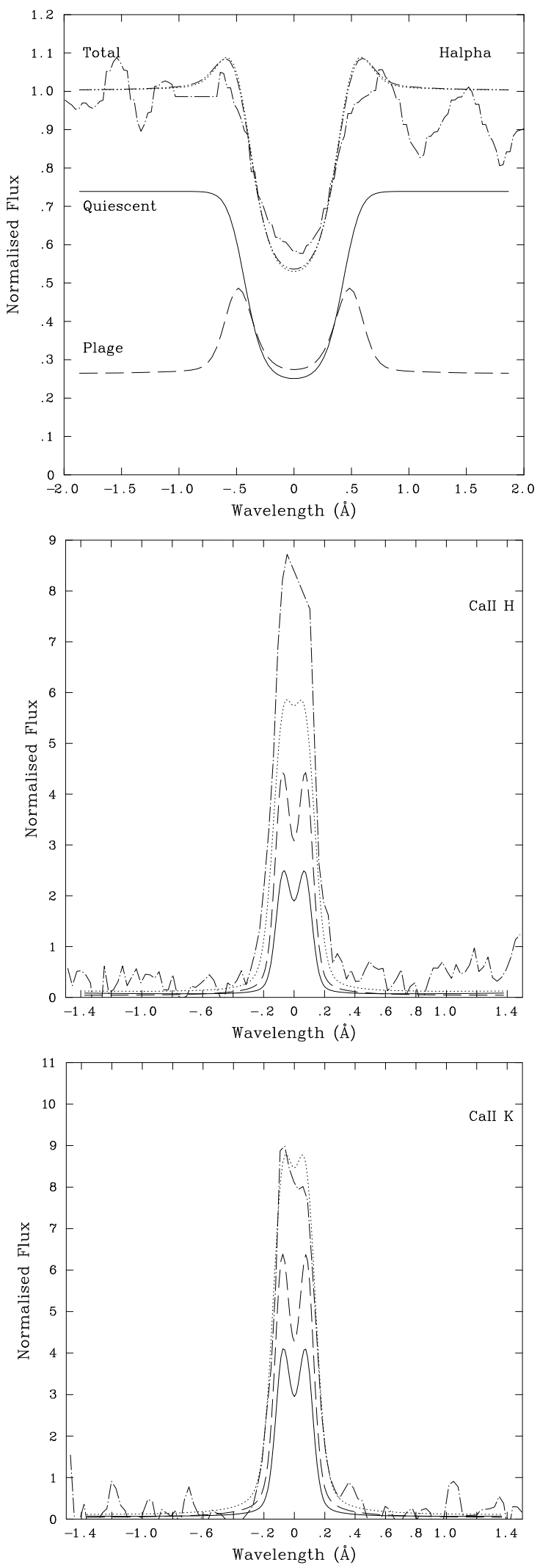

Fig. 8. In this figure, we show our best fits to the $\mathrm{H}_{\alpha}$, CaII H and CaII $\mathrm{K}$ lines for Gl 49. The quiescent region contribution is shown as a continuous line, the plage contribution as a dashed line, the total theoretical profiles for the lower solution as a dotted line, the total theoretical profiles for the higher solution as a dashed-double dotted line, and the observed profiles as a dashed-dotted line. 


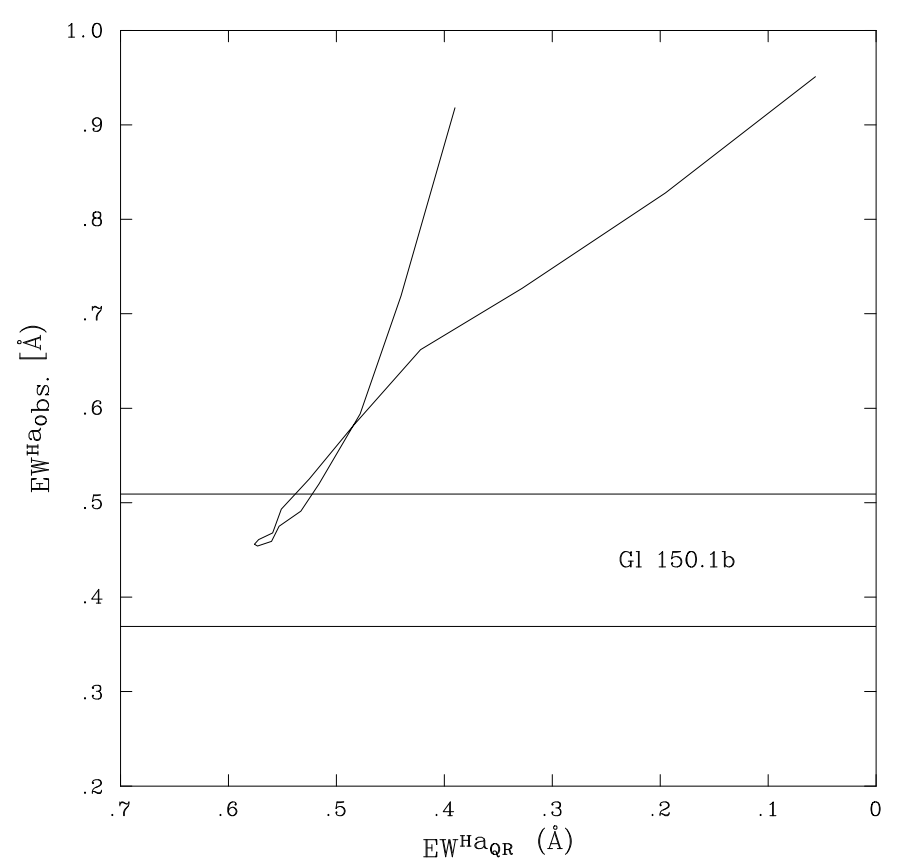

Fig. 9. We show $E W_{\mathrm{Obs}}^{\mathrm{H}_{\alpha}}$ as a function of $E W_{\mathrm{QR}}^{\mathrm{H}_{\alpha}}$ for $\mathrm{Gl} 150.1 \mathrm{~B}$ for the observed value of the mean $\mathrm{CaII} H \& \mathrm{~K}$ equivalent width. The range of our observations of the $\mathrm{H}_{\alpha}$ equivalent width for Gl 150.1B is also indicated as the two horyzontal lines that represent the $\pm 3 \sigma$ limit on the $\mathrm{H}_{\alpha}$ equivalent width measurement. One can see that there is only one solution possible for Gl 150.1B.

In Fig. 10, we show the theoretical and observed profiles. The total theoretical profiles were convolved with the ELODIE instrumental profile and a Gaussian of $F W H M 2.2 \mathrm{~km} \mathrm{~s}^{-1}$ $(v \sin i)$. In the case of $\mathrm{H}_{\alpha}$, the fit is reasonable except in the wings. As noted above (see also Sect. 14), this is probably due to blends with photospheric absorption lines. For our model, the entire profile is dominated by quiescent regions.

For the CaII lines, the CaII H line profile is too noisy for comparison to the models. The agreement between the model and the observed profile of the CaII K line is reasonable. As in other intermediate-activity stars studied here, the theoretical CaII line profiles show a weak central absorption which is indicative of a rather high chromospheric optical depth. The CaII line profiles also are largely dominated by the contribution from active regions.

The observed CaII line equivalent widths are of the same order for Gl 150.1B as for Gl 2 and Gl 205. However, the resulting models differ slightly; the column mass at the transition region is slightly larger in Gl 2 and Gl 205 than in Gl 150.1B (both for quiescent and active regions). This produces slightly lower surface fluxes in the CaII lines for Gl 150.1B. Also, for of Gl 2 and Gl 205, the $\mathrm{H}_{\alpha}$ profile is also more filled-in or slightly in emission, whereas in the case of G1 $150.1 \mathrm{~B}$ it shows a stronger absorption. This difference in the chromospheric pressure is compensated by a larger filling factor for Gl 150.1B. Nevertheless, as we shall see in Sect. 14, the results for these three stars are rather homogeneous.

For the error domain of the CaII mean equivalent width the problem is that we have only an estimate of the CaII mean equivalent width with no evaluation of the error; this latter value being too large. We are therefore unable to estimate the error in our models.

As far as the uncertainty in $\mathrm{H}_{\alpha}$ is concerned, we consider our solution for the CaII mean equivalent width of $-1.4 \AA$. We
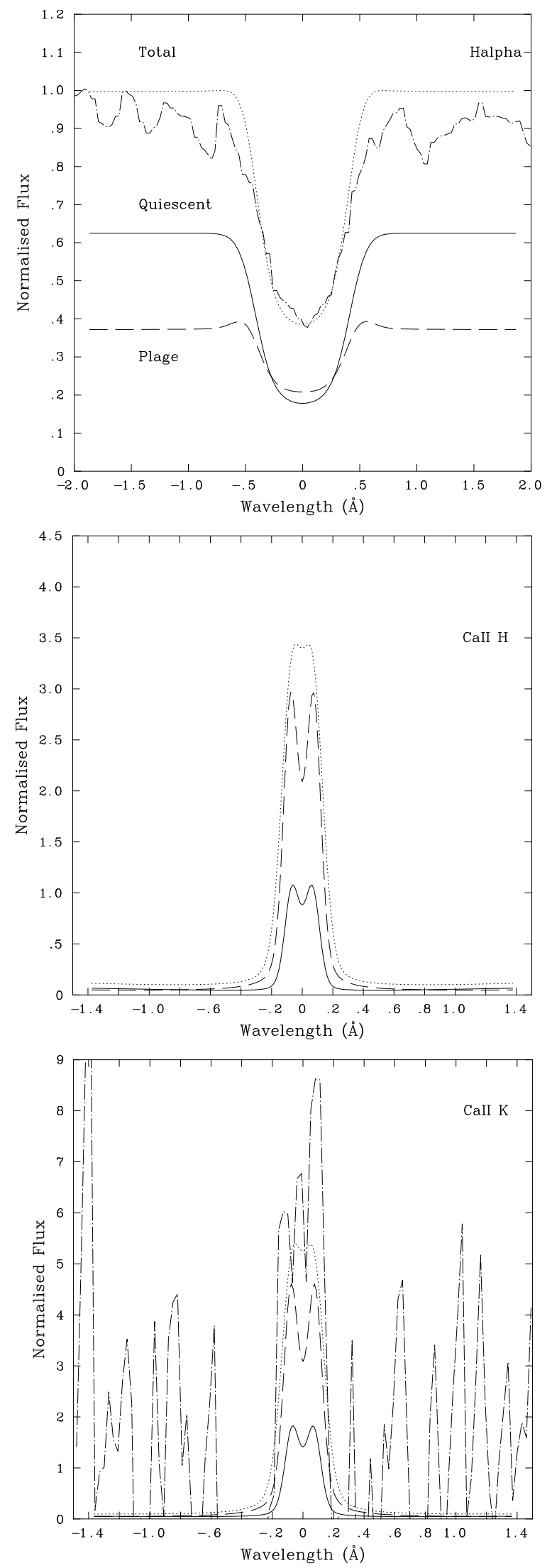

Fig. 10. In this figure we show our best fits to the $\mathrm{H}_{\alpha}$ and CaII $\mathrm{K}$ lines for G1 150.1B. The quiescent region contribution is shown as a continuous line, the plage contribution as a dashed line, the total theoretical profiles as a dotted line, and the observed profiles as a dashed-dotted line. 
then consider the $\pm \sigma$ domain in $\mathrm{H}_{\alpha}$. We have two solutions for the upper value to $\mathrm{H}_{\alpha}$ of $0.462 \AA$ (see Fig. 9). From these two solutions, we estimate the uncertainties in our model due to $\mathrm{H}_{\alpha}$. We find that the resulting uncertainties in the model are $\delta \mathrm{H}_{\alpha}^{\mathrm{QR}}= \pm 0.007 \AA, \delta \mathrm{H}_{\alpha}^{\text {Plage }}= \pm 0.19 \AA, \delta \mathrm{CaII}^{\mathrm{QR}}= \pm 0.17 \AA$, $\delta \mathrm{CaII}^{\text {Plage }}= \pm 0.85 \AA, \delta f / f= \pm 40 \%, \delta \log (M)_{\mathrm{QR}}= \pm 0.082$, and $\delta \log (M)_{\text {Plage }}= \pm 0.067$. These values are relatively small compared to other stars, except for the filling factor.

\section{Model chromosphere for GI 229}

Gl 229 has a relatively small CaII mean equivalent width (-1.01 $\AA$, Table 1). This value was taken from Paper VI, because the CaII lines are not detected in our spectra, and represents the mean of 22 single measurements. The $\mathrm{H}_{\alpha}$ equivalent width is the largest observed for our solar metallicity M1 dwarfs $(0.497 \AA)$. These observations of $\mathrm{H}_{\alpha}$ and CaII line were obtained at different times. These equivalent widths indicate that this is a star with a strong absorption $\mathrm{H}_{\alpha}$ profile for quiescent regions. However, as we see below, the active region profile is filled in and this is therefore another star of intermediate-activity (with a filled-in global $\mathrm{H}_{\alpha}$ profile).

We show $E W_{\text {Obs. }}^{\mathrm{H}_{\alpha}}$ as a function of $E W_{\mathrm{QR}}^{\mathrm{H}_{\alpha}}$ for the observed value of the CaII mean equivalent width in Fig. 11. One can see from this curve that there is only one most likely solution, which corresponds to the minimum of the curve. The minimum $E W_{\text {Obs. }}^{\mathrm{H}_{\alpha}}$ given by our models is $0.502 \AA$ : there is a difference of only $0.005 \AA$ with the observed value. This solution corresponds to CaII mean equivalent widths of $-0.477 \AA$ and $-2.385 \AA$ for the quiescent regions and active regions, respectively. The corresponding plage filling factor is $28 \%$. The values of the CaII mean equivalent widths of the quiescent regions and active regions are significantly smaller than for other intermediate-activity stars (e.g., Gl 2 and Gl 205).

In Fig. 12, we show the theoretical and observed profiles for our best-fit solution The total theoretical profiles were convolved with the ELODIE instrumental profile and a Gaussian of $F W H M 1.3 \mathrm{~km} \mathrm{~s}^{-1}(v \sin i)$. There is reasonable agreement with the $\mathrm{H}_{\alpha}$ profile except in the wings. The fit is reasonable at the line center. In the case of Gl 229, the $\mathrm{H}_{\alpha}$ profile is largely dominated by the contribution of quiescent regions. In Fig. 12, we can see that the main contribution to the CaII lines still originates in the active regions, as in most other stars.

For the error domain of the CaII mean equivalent width, we consider an uncertainty of $\pm 0.1 \AA$. The value of the CaII mean equivalent width given in Table 1 is the average of 22 measurements. The uncertainty that we choose here corresponds to a high signal-to-noise ratio spectrum (see Houdebine 2009b, Paper XII). We find solutions for both the lower value and upper value of the CaII mean equivalent width. From these two solutions, we estimate the uncertainties in our model. We find that the resulting uncertainties in the model are $\delta \mathrm{H}_{\alpha}^{\mathrm{QR}}= \pm 0.007 \AA$, $\delta \mathrm{H}_{\alpha}^{\text {Plage }}= \pm 0.048 \AA, \delta \mathrm{CaII}{ }^{\mathrm{QR}}= \pm 0.044 \AA, \delta \mathrm{CaII}^{\text {Plage }}= \pm 0.22 \AA$, $\delta f / f= \pm 0.5 \%, \delta \log (M)_{\mathrm{QR}}= \pm 0.025$, and $\delta \log (M)_{\text {Plage }}=$ \pm 0.019 . These figures are rather small, and is a consequence of the small uncertainty in the CaII $E W$.

As far as the uncertainty in $\mathrm{H}_{\alpha}$ is concerned, we consider our solution for the CaII mean equivalent width of $-1.01 \AA$. We then consider the $\pm \sigma$ domain in $\mathrm{H}_{\alpha}$. We have two solutions for the upper value of $\mathrm{H}_{\alpha}$ of $0.514 \AA$. From these two solutions, we estimate the uncertainties in our model related to $\mathrm{H}_{\alpha}$. We find that the resulting uncertainties in the model

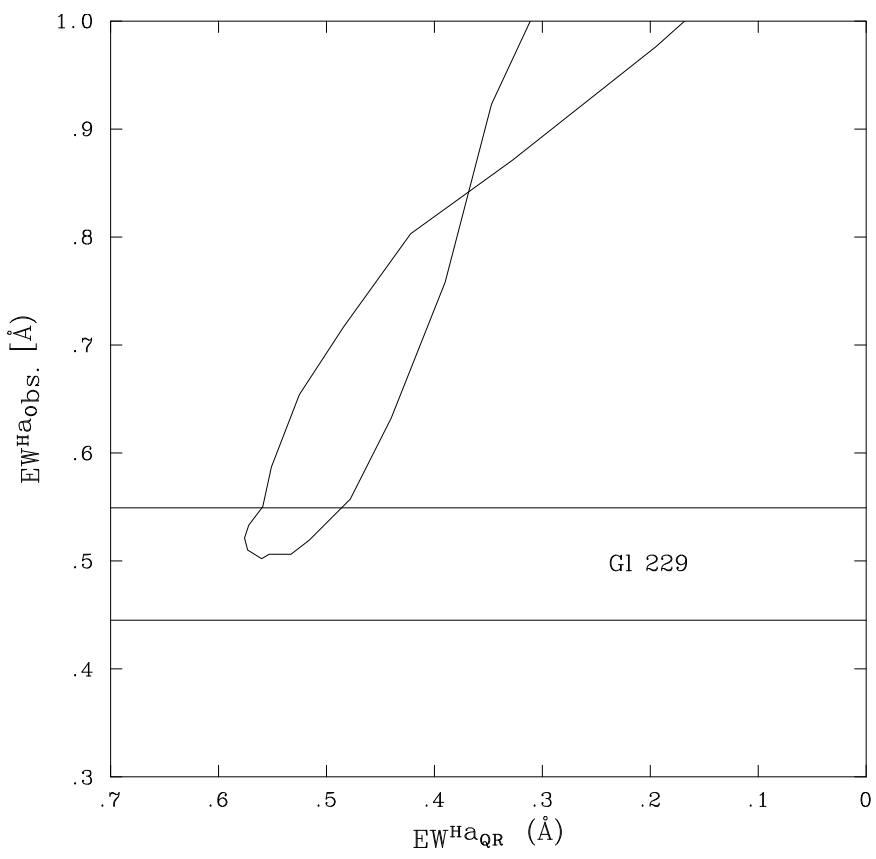

Fig. 11. We show $E W_{\text {Obs. }}^{\mathrm{H}_{\alpha}}$ as a function of $E W_{\mathrm{QR}}^{\mathrm{H}_{\alpha}}$ for Gl 229 for the mean CaII H \& K equivalent width of $-1.01 \AA$. The range of our measurements of $\mathrm{H}_{\alpha}$ equivalent width for Gl 229 is also indicated by the two horyzontal lines that represent the $\pm 3 \sigma$ limit to the $\mathrm{H}_{\alpha}$ equivalent-width measurement. One can see that there is only one most likely solution.

are: $\delta \mathrm{H}_{\alpha}^{\mathrm{QR}}= \pm 0.026 \AA, \delta \mathrm{H}_{\alpha}^{\mathrm{Plage}}= \pm 0.15 \AA, \delta \mathrm{CaII} \mathrm{IR}^{\mathrm{QR}}= \pm 0.15 \AA$, $\delta$ CaII ${ }^{\text {Plage }}= \pm 0.75 \AA, \delta f / f= \pm 41 \%, \delta \log (M)_{\mathrm{QR}}= \pm 0.096$ and $\delta \log (M)_{\text {Plage }}= \pm 0.085$. These values are of the same order as those found for Gl205, and yield a total uncertainty that is one of the best for our stars (Table 3). in particular the total uncertainty in the column mass is fairly small and therefore restricts the possible solutions for G1 229 considerably.

\section{Model chromosphere for GI 526}

G1526 is another interesting low-activity star with a mean CaII equivalent width of $-0.491 \AA$. Its $\mathrm{H}_{\alpha}$ equivalent width of $0.463 \AA$ is much larger than that of GJ $1010 \AA$, which indicates that it has a higher activity level. These values of the equivalent widths were taken from the compilation of Paper VI. The CaII mean equivalent width is the average of 38 single measurements. During our observing campaign with ELODIE, we did not observe this star but we still analyse the data for this star here because it is one of the few low-activity dM1 star of solar metallicity. Most stars of solar metallicity have a higher activity level (Papers VI and VII). We emphasize that the $\mathrm{H}_{\alpha}$ and CaII line observations used here were not obtained simultaneously. As the uncertainty in the $\mathrm{H}_{\alpha}$ equivalent width, we assumed a typical value of $\pm 0.02 \AA$ (Paper VI). This uncertainty is mostly due to the difficulty in determining the adjacent continuum level.

We show $E W_{\text {Obs. }}^{\mathrm{H}_{\alpha}}$ as a function of $E W_{\mathrm{QR}}^{\mathrm{H}_{\alpha}}$ for the observed value of the CaII mean equivalent width in Fig. 13. One can see from this curve that there is only one most likely solution, which corresponds to the minimum value of the curve. The minimum $E W_{\text {Obs. }}^{\mathrm{H}_{\alpha}}$ given by our models is $0.506 \AA$, which is significantly above the average measurement for G1526 but remains within the uncertainty interval. This solution infers low CaII mean equivalent widths of $-0.203 \AA$ and $-1.015 \AA$ for 

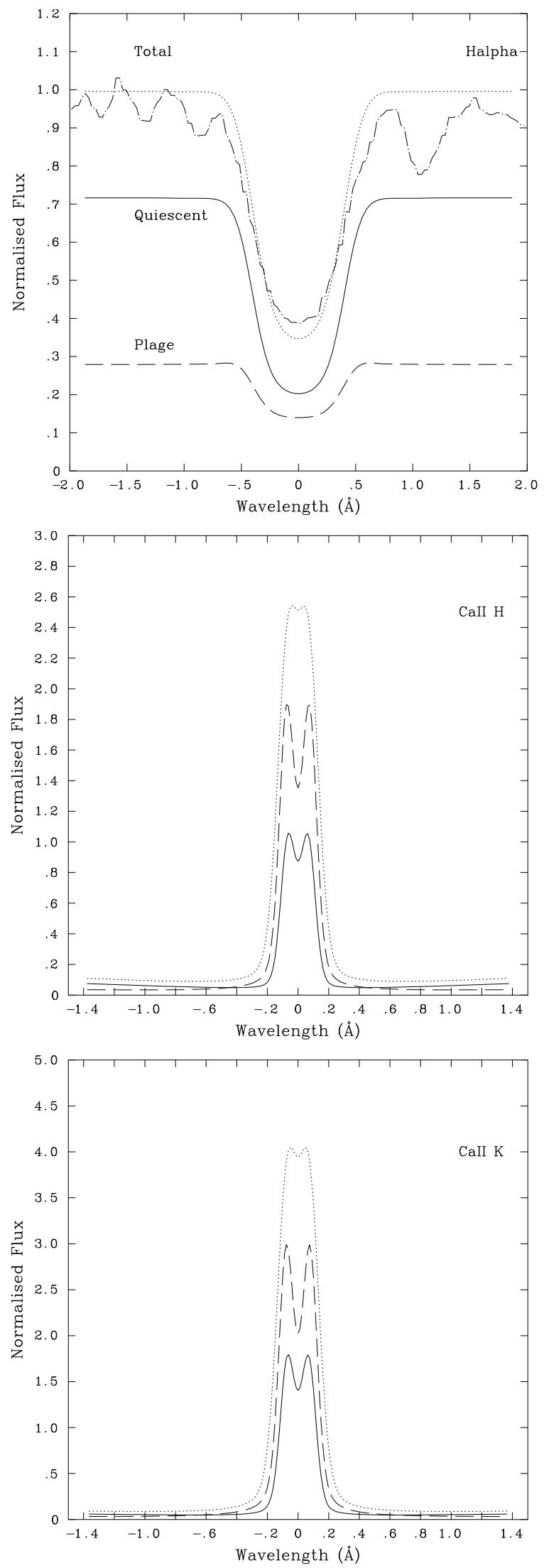

Fig. 12. In this figure we show our best fits to the $\mathrm{H}_{\alpha}$ profile and the $\mathrm{CaII} \mathrm{H}$ and CaII K theoretical profiles for Gl 229. The quiescent region contribution is shown as a continuous line, the plage contribution as a dashed line, the total theoretical profiles as a dotted line, and the observed profile as a dashed-dotted line.

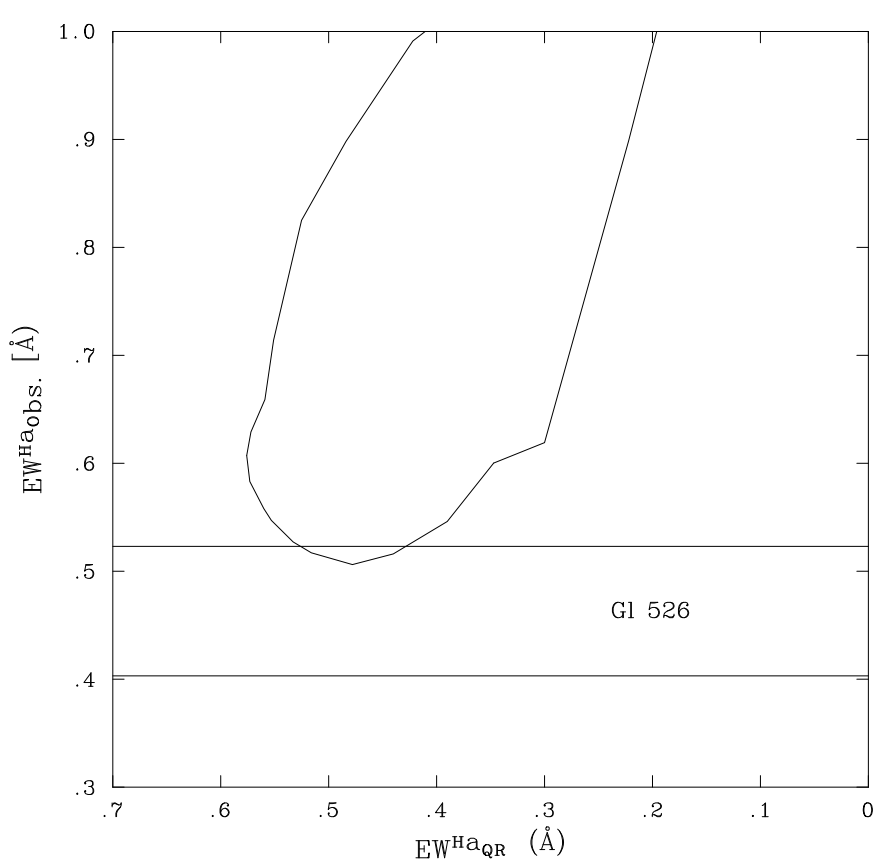

Fig. 13. We show $E W_{\text {Obs. }}^{\mathrm{H}_{\alpha}}$ as a function of $E W_{\mathrm{QR}}^{\mathrm{H}_{\alpha}}$ for $\mathrm{Gl} 526$ for the mean CaII H \& K equivalent width of $-0.491 \AA$. The range of our measurements of the $\mathrm{H}_{\alpha}$ equivalent width for Gl 526 is also indicated as the two horyzontal lines that represent the $\pm 3 \sigma$ limit on the $\mathrm{H}_{\alpha}$ equivalent width measurement. One can see that there is one most likely solution possible within measurement errors.

the quiescent regions and active regions respectively. The corresponding plage filling factor is $36 \%$. We note that for the low activity stars GJ 1010A and Gl526, we find filling factors for plages that are of the same order as those found for higher activity solar-metallicity dM1 stars, including the most active ones, Gl 49 and G192-11A. Therefore, even in rather lowactivity stars, the plages represent a significant fraction of the stellar surface.

In Fig. 14, we show the theoretical profiles for our best fit solution. The total theoretical profiles were convolved with the ELODIE instrumental profile to allow comparison with other observations. The $\mathrm{H}_{\alpha}$ profile remains dominated by the contribution of quiescent regions, whereas the main contribution to the CaII lines comes from the active regions. One can see that the relative depth of the central absorption in the CaII line profiles diminishes significantly with the activity level. Eventually, it totally disappears as for the quiescent regions of GJ 1010A (Fig. 6). At these low levels of activity, the CaII lines are effectively optically thin (Paper VI).

For the CaII mean equivalent width, we assume an uncertainty of $\pm 0.1 \AA$. The value of the CaII mean equivalent width given in Table 1 is the average of 38 measurements (Paper VI). The uncertainty that we choose here was measured in a high signal-to-noise ratio spectrum (see Houdebine 2009b, Paper XII). We find solutions for the lower value and the upper values of the CaII mean equivalent width. From these two solutions, we estimate the uncertainties in our model. We find that the resulting uncertainties in the model are $\delta \mathrm{H}_{\alpha}^{\mathrm{QR}}= \pm 0.019 \AA$, $\delta \mathrm{H}_{\alpha}^{\text {Plage }}= \pm 0.008 \AA, \delta$ CaII ${ }^{\mathrm{QR}}= \pm 0.027 \AA, \delta \mathrm{CaII}^{\text {Plage }}= \pm 0.14 \AA$, $\delta f / f= \pm 3.5 \%, \delta \log (M)_{\mathrm{QR}}= \pm 0.050$ and $\delta \log (M)_{\text {Plage }}=$ \pm 0.044 . These figures are rather small, due to the small uncertainty on the CaII $E W$. 

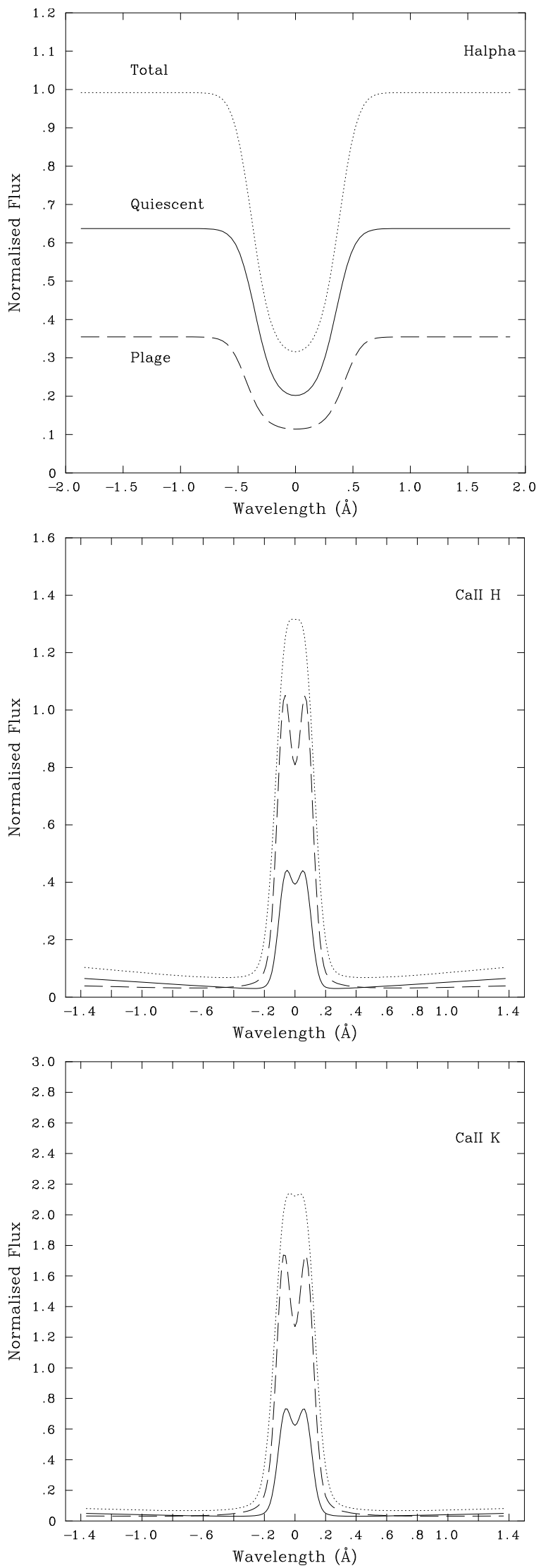

Fig. 14. We show the $\mathrm{H}_{\alpha}$, CaII $\mathrm{H}$ and CaII $\mathrm{K}$ line profiles for G1 526. The quiescent region contribution is shown as a continuous line, the plage contribution as a dashed line and the total theoretical profiles as a dotted line.

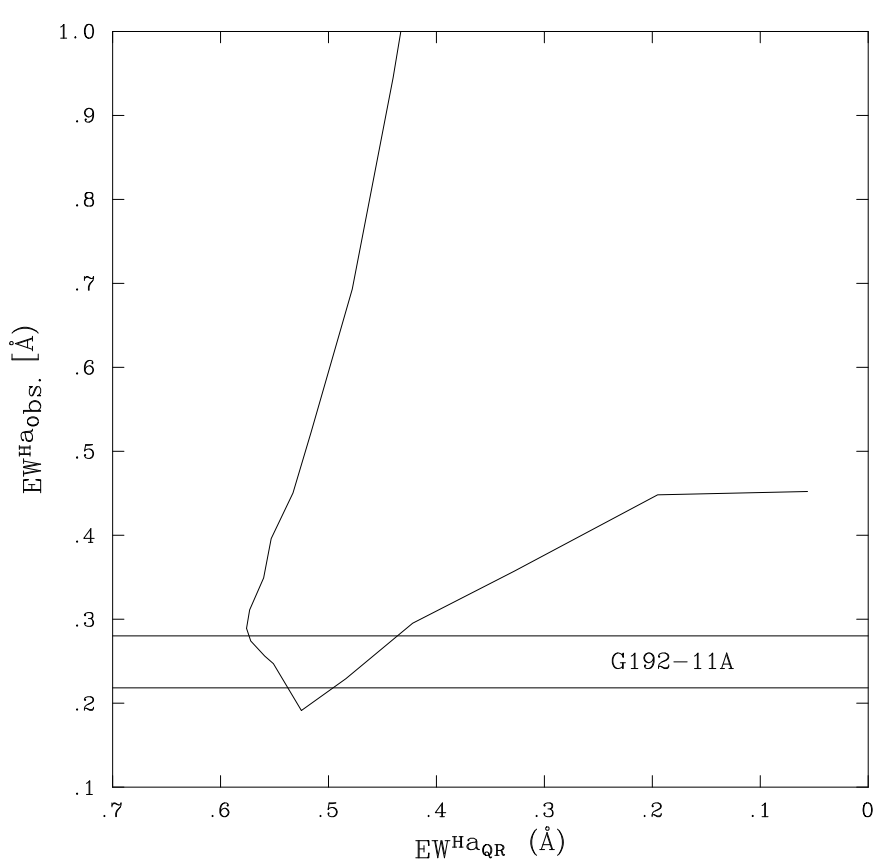

Fig. 15. We show $E W_{\text {Obs. }}^{\mathrm{H}_{\alpha}}$ as a function of $E W_{\mathrm{QR}}^{\mathrm{H}_{\alpha}}$ for G192-11A for the mean CaII $\mathrm{H} \& \mathrm{~K}$ equivalent width of $-2.41 \AA$. The range of our measurements of the $\mathrm{H}_{\alpha}$ equivalent width for G192-11A is also indicated as the two horyzontal lines that represent the $\pm 3 \sigma$ limit on the $\mathrm{H}_{\alpha}$ equivalent width measurement. One can see that there are two solutions possible.

As far as the uncertainty in $\mathrm{H}_{\alpha}$ is concerned, we consider our solution for the CaII mean equivalent width of $-0.491 \AA$. We then consider the uncertainty interval of $\pm 3 \sigma$ for $\mathrm{H}_{\alpha}$. We have two solutions for the upper limit to $\mathrm{H}_{\alpha}$ of $0.523 \AA$. From these two solutions, we estimate the uncertainties in our model due to $\mathrm{H}_{\alpha}$. We find that the resulting uncertainties in the model are $\delta \mathrm{H}_{\alpha}^{\mathrm{QR}}= \pm 0.037 \AA, \delta \mathrm{H}_{\alpha}^{\text {Plage }}= \pm 0.037 \AA, \delta \mathrm{CaII}{ }^{\mathrm{QR}}= \pm 0.078 \AA$, $\delta \mathrm{CaII}^{\text {Plage }}= \pm 0.39 \AA, \delta f / f= \pm 43 \%, \delta \log (M)_{\mathrm{QR}}= \pm 0.099$, and $\delta \log (M)_{\text {Plage }}= \pm 0.088$. These values are of the same order as those found for G1229, and yield a total uncertainty that is one of the lowest for our stars (Table 3), especially in terms of equivalent widths.

\section{Model chromosphere for G192-11A}

With a CaII mean equivalent width of $-2.41 \AA$, G192-11A is the most active of the nine M1 dwarfs discussed in this paper. The $\mathrm{H}_{\alpha}$ and CaII equivalent widths for this star are very similar to those of Gl 49. In Paper VI, G192-11A was not the most active M1 dwarf reported; G1 763 is a bit more active with a CaII mean equivalent width of $-2.47 \AA$. With a CaII mean equivalent width of $-2.43 \AA$, Gl 140A is also quite active. These stars are the most active M1 dwarfs, and are almost as active as dM1e stars: for instance the CaII mean equivalent width of the dM1e star G1 867A, a near solar metallicity star, is only $-4.2 \AA$. The intermediateactivity stars have even larger CaII mean equivalent widths than the M1e subdwarf G1 781 (-1.71 $\AA$ ).

From Eq. (6), we show $E W_{\text {Obs. }}^{\mathrm{H}_{\alpha}}$ as a function of $E W_{\mathrm{QR}}^{\mathrm{H}_{\alpha}}$ for the observed values of the CaII mean equivalent width in Fig. 15. This curve is similar to that of Gl49. For G192-11A, the minimum $E W_{\text {Obs. }}^{\mathrm{H}_{\alpha}}$ is only $-0.191 \AA$. We find two solutions for G192-11A : the lower solution infers a plage filling factor of 
$29 \%$ and the higher solution a plage filling factor of only $9 \%$. Their respective CaII mean equivalent width for quiescent regions are $-1.12 \AA$ and $-1.80 \AA$. This latter value seems a bit high for quiescent regions, especially when compared to those of Gl 205 and Gl 2. The filling factor derived for the lower solution is also more consistent with the values derived for the other stars. For these reasons, we again prefer the lower solution. In any case, the uncertainty in this model includes the higher solution (Table 3).

In Fig. 16, we show the theoretical and observed profiles for the two solutions. The total theoretical profiles were convolved with the ELODIE instrumental profile and a Gaussian of FWHM $1.4 \mathrm{~km} \mathrm{~s}^{-1}(v \sin i)$. One can see that there is very little difference between the $\mathrm{H}_{\alpha}$ profiles for the two solutions, as in the case of Gl 49. We obtain theoretical profiles that are very similar to those of Gl 49. The observed profiles for the two stars are also quite similar. In the case of $\mathrm{H}_{\alpha}$, the fit is reasonable except at the line center, where the theoretical model shows a stronger absorption than the observed profile. The theoretical profiles also exhibit weak emission wings. As for the previous stars, if we do not detect these weak emission wings in the observed profile, this is because of probable blends in the wings of $\mathrm{H}_{\alpha}$. For G192-11A, the contribution of the plage regions to the $\mathrm{H}_{\alpha}$ profile is a bit larger than in G149.

We can see in Fig. 16 that the plage contribution to the CaII lines is almost twice as large as the contribution from quiescent regions, although the contribution from quiescent regions is not negligible. In this figure, we show only the lower solution for the CaII profiles. Our model again slightly underestimates the $\mathrm{CaII} \mathrm{H}$ flux. The observed profiles are flattened at line center as in Gl49, but do not exhibit a central absorption. The results for G192-11A are very similar to those of G1 49.

For the uncertainty in the CaII mean equivalent width, we consider the solution for the lower value of the CaII mean equivalent width of $-2.25 \AA$. We also find a solution for the maximum value of $-2.57 \AA$. From these two solutions, we estimate the uncertainties in our model. We find that the resulting uncertainties in the model are $\delta \mathrm{H}_{\alpha}^{\mathrm{QR}}= \pm 0.011 \AA, \delta \mathrm{H}_{\alpha}^{\text {Plage }}= \pm 0.39 \AA$, $\delta \mathrm{CaII}^{\mathrm{QR}}= \pm 0.19 \AA, \delta \mathrm{CaII}^{\mathrm{Plage}}= \pm 0.97 \AA, \delta f / f= \pm 18 \%$, $\delta \log (M)_{\mathrm{QR}}= \pm 0.0561$ and $\delta \log (M)_{\text {Plage }}= \pm 0.052$. These values are of the same order as those of Gl 49, which has a similar activity level. We also list these values in Table 3 .

As far as the uncertainty in $\mathrm{H}_{\alpha}$ is concerned, we consider our lower solution for the CaII mean equivalent width of $-2.41 \AA$. We then consider the $\pm \sigma$ uncertainty in $\mathrm{H}_{\alpha}$. We have two solutions for the upper value of $\mathrm{H}_{\alpha}$ of $0.259 \AA$ and the lower value of $0.239 \AA$. We choose the lower solution in each case, in a similar way to our final solution for this star, and because the filling factors are in closer agreement with other stars. This is one of few stars in our sample for which we find solutions for the lower and upper values in both $\mathrm{H}_{\alpha}$ and CaII lines. From these two solutions, we estimate the uncertainties in our model due to $\mathrm{H}_{\alpha}$. We find that the resulting uncertainties in the model are $\delta \mathrm{H}_{\alpha}^{\mathrm{QR}}= \pm 0.008 \AA, \delta \mathrm{H}_{\alpha}^{\text {Plage }}= \pm 0.19 \AA, \delta \mathrm{CaII}^{\mathrm{QR}}= \pm 0.13 \AA$, $\delta \mathrm{CaII}^{\text {Plage }}= \pm 0.64 \AA, \delta f / f= \pm 7 \%, \delta \log (M)_{\mathrm{QR}}= \pm 0.031$ and $\delta \log (M)_{\text {Plage }}= \pm 0.036$. We also list these values in Table 3 . These values are a bit smaller than those found for G149, and yield a total uncertainty that is one of the lowest for our stars (Table 3). In particular, the total uncertainty in the column mass is fairly small and therefore restricts the possible solutions considerably.
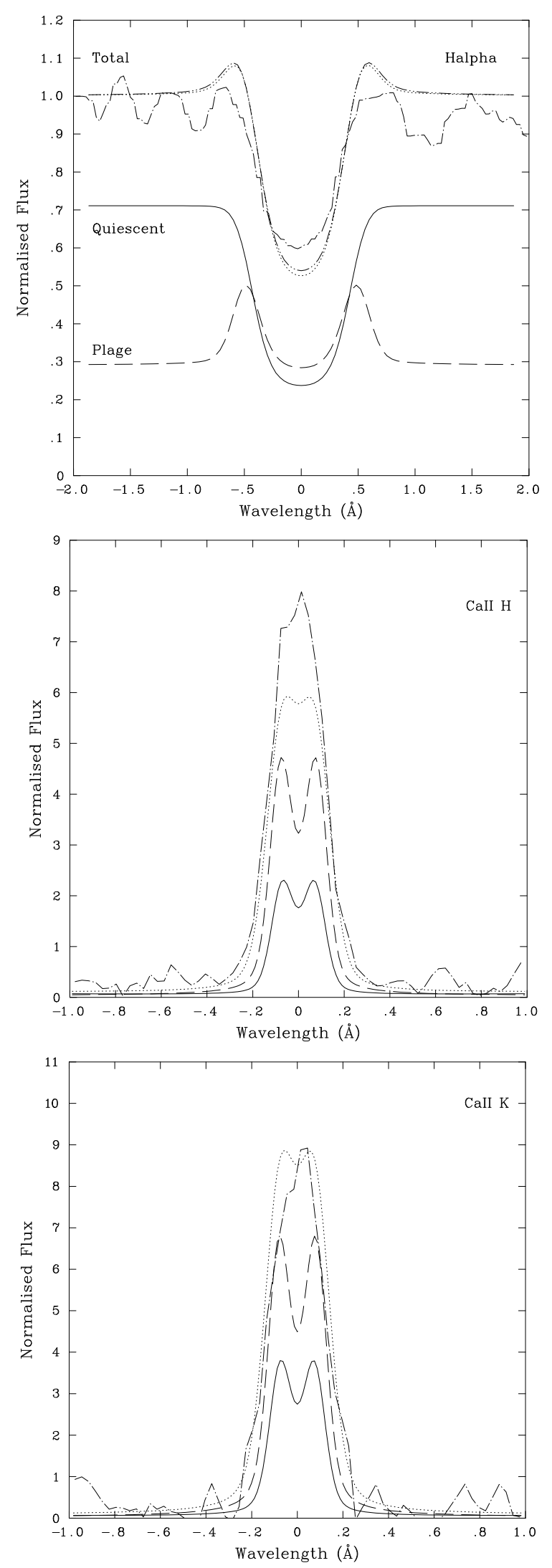

Fig. 16. We show our best fits to the $\mathrm{H}_{\alpha}$, CaII $\mathrm{H}$ and CaII $\mathrm{K}$ lines for G192-11A. The quiescent region contribution is shown as a continuous line, the plage contribution as a dashed line, the total theoretical profiles for the lower solution as a dotted line, the total theoretical profiles for the higher solution as a dashed-double dotted line, and the observed profiles as a dashed-dotted line. 


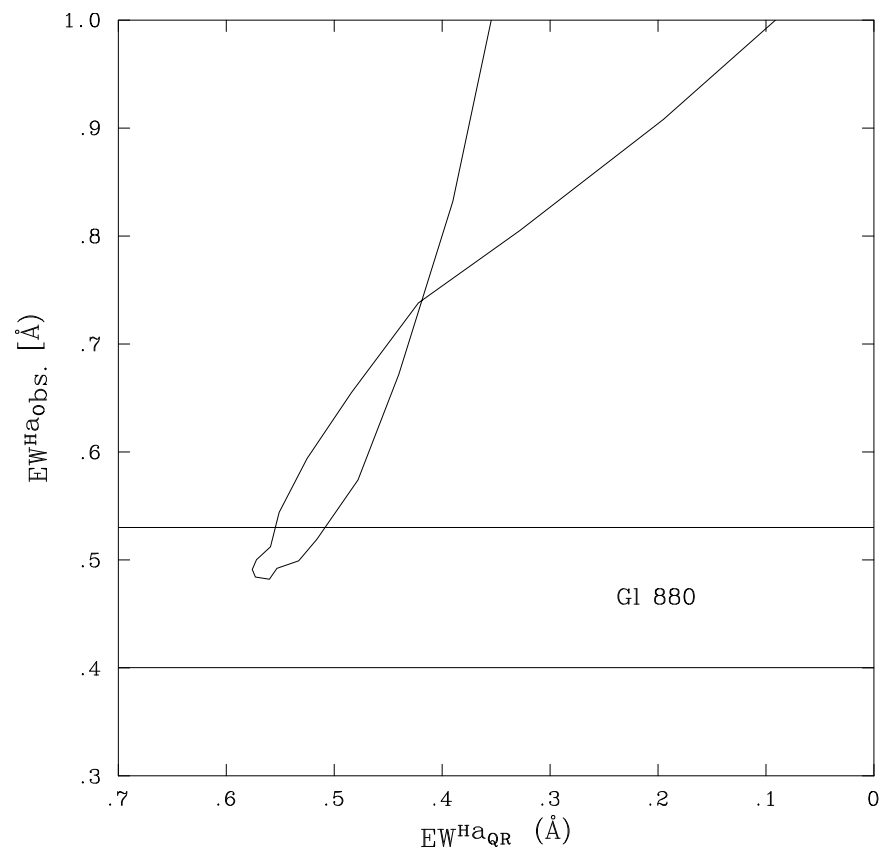

Fig. 17. We show $E W_{\text {Obs. }}^{\mathrm{H}_{\alpha}}$ as a function of $E W_{\mathrm{QR}}^{\mathrm{H}_{\alpha}}$ for $\mathrm{Gl} 880$ for the mean CaII H \& K equivalent width of $-1.19 \AA$. The range of our measurements of the $\mathrm{H}_{\alpha}$ equivalent width for Gl 880 is also indicated as the two horyzontal lines that represent the $\pm 3 \sigma$ limit on the $\mathrm{H}_{\alpha}$ equivalent width measurement. One can see that there is only one most likely solution.

\section{Model chromosphere for GI 880}

In Paper VI, we measured a mean CaII equivalent width of $-1.19 \AA$ for $\mathrm{Gl} 880$. The $\mathrm{H}_{\alpha}$ equivalent width, $0.465 \AA$ (Table 1), is also large for this star. This indicates that this star is of intermediate-activity.

We compute $E W_{\text {Obs. }}^{\mathrm{H}_{\alpha}}$ as a function of $E W_{\mathrm{QR}}^{\mathrm{H}_{\alpha}}$ for the mean observed value of the CaII lines. We show the resulting curve in Fig. 17. We find one solution for this star, which corresponds to the minimum of the curve in Fig. 17. The minimum predicted $E W_{\text {Obs }}^{\mathrm{H}_{\alpha}}$ is $0.482 \AA$, which is close to the value $0.465 \AA$ observed.

The theoretical CaII mean equivalent width for this model, is $-0.477 \AA$ and $-2.385 \AA$ for quiescent regions and active regions, respectively. We obtain a plage filling factor of $37 \%$ for these values. This is one of the largest filling factors that we have found for dM1 stars. All these values are comparable to those found for Gl 150.1B. We note that this latter star has a CaII mean equivalent width of $-1.4 \AA$, close to that of Gl 880 .

We show in Fig. 18 the theoretical and observed profiles. The total theoretical profiles were convolved with the ELODIE instrumental profile and a Gaussian of FWHM $1.3 \mathrm{~km} \mathrm{~s}^{-1}(v \sin i)$. In the case of $\mathrm{H}_{\alpha}$, the observations can be rather well reproduced, except in the red wing. Here again, the whole profile is dominated by quiescent regions.

For the CaII lines, the agreement between the model and the observed profiles is acceptable, taking into account the noise in the spectra. The observed CaII K line shows a possible central absorption, but not the $\mathrm{CaII} \mathrm{H}$ line. The $\mathrm{CaII}$ line profiles are also largely dominated by the contribution from active regions.

The model of Gl 880 differs slightly from that of Gl 150.1B; the column mass at the transition region is slightly lower in Gl 880 than in Gl150.1B (both for quiescent and active regions, Table 4). This results in slightly lower surface fluxes in the CaII lines in the case of Gl 880. This difference in the
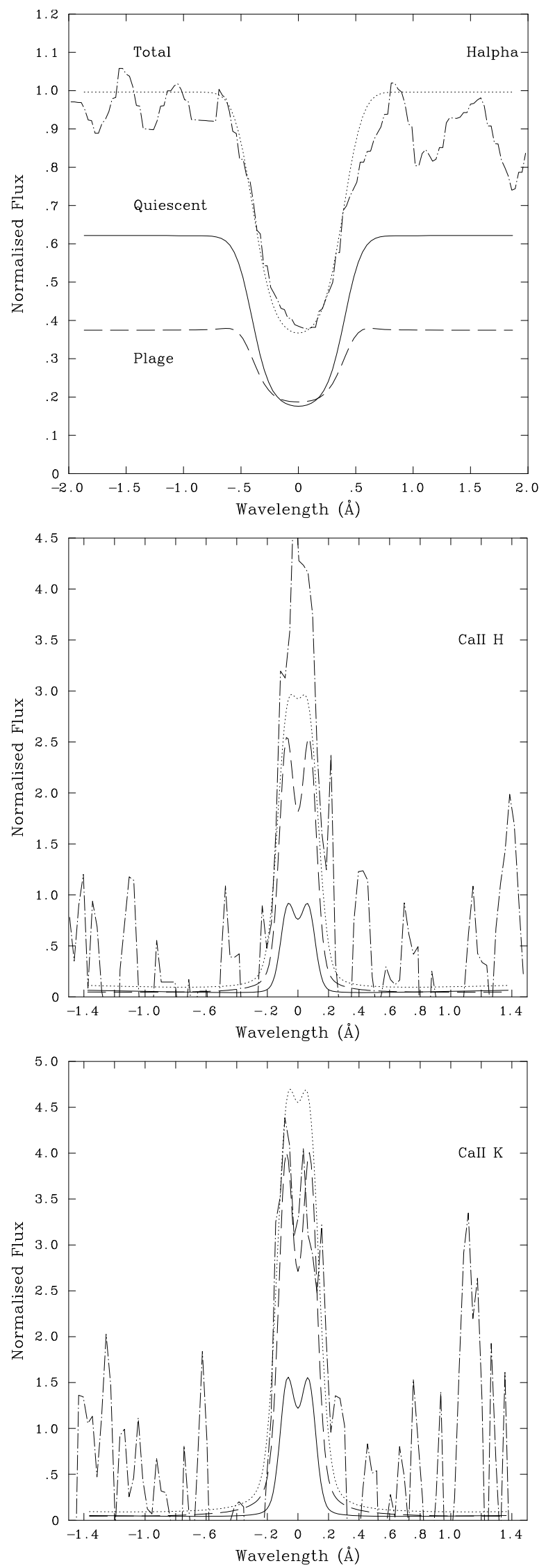

Fig. 18. We show our best-fit models for the $\mathrm{H}_{\alpha}$, CaII $\mathrm{H}$ and CaII $\mathrm{K}$ lines for Gl 880 . The quiescent region contribution is shown as a continuous line, the plage contribution as a dashed line, the total theoretical profiles as a dotted line, and the observed profiles as a dashed-dotted line. 
Table 4. Column mass at the transition region and spectral line equivalent widths for quiescent regions and plages for the near-solar metallicity stars of this paper.

\begin{tabular}{cccccccc}
\hline \hline Star & $f$ & $\begin{array}{c}\text { Call } E W \\
\text { Quiescent regions } \\
(\AA)\end{array}$ & $\begin{array}{c}\text { CaII } E W \\
\text { Active regions } \\
(\AA)\end{array}$ & $\begin{array}{c}\mathrm{H}_{\alpha} E W \\
\text { Quiescent regions } \\
(\AA)\end{array}$ & $\begin{array}{c}\mathrm{H}_{\alpha} E W \\
\text { Active regions } \\
(\AA)\end{array}$ & $\begin{array}{c}\log (\text { column mass) } \\
\text { Quiescent regions } \\
\left(\mathrm{g} \mathrm{cm}^{-2}\right)\end{array}$ & $\begin{array}{c}\text { log(column mass) } \\
\text { Active regions } \\
\left(\mathrm{g} \mathrm{cm}^{-2}\right)\end{array}$ \\
\hline G12 & $9 \pm 2$ & -0.967 & -4.84 & 0.559 & -0.251 & -4.450 & -4.026 \\
GJ 1010A & $32 \pm 62$ & -0.081 & -0.405 & 0.300 & 0.552 & -5.200 & -4.604 \\
G1 49 & $26 \pm 29$ & -1.17 & -5.83 & 0.551 & -0.591 & -4.299 & -3.886 \\
G1 150.1B & $37 \pm 40$ & -0.564 & -2.82 & 0.573 & 0.253 & -4.500 & -4.072 \\
G1 205 & $15 \pm 22$ & -0.884 & -4.42 & 0.566 & -0.133 & -4.375 & -3.964 \\
G1 229 & $28 \pm 42$ & -0.477 & -2.38 & 0.560 & 0.349 & -4.550 & -4.111 \\
G1 526 & $36 \pm 46$ & -0.203 & -1.02 & 0.478 & 0.557 & -4.800 & -4.337 \\
G192-11A & $29 \pm 25$ & -1.12 & -5.59 & 0.553 & -0.458 & -4.311 & -3.903 \\
G1 880 & $37 \pm 27$ & -0.477 & -2.38 & 0.560 & 0.349 & -4.550 & -4.111 \\
\hline
\end{tabular}

chromospheric pressure is due to the lower observed CaII $E W$, because these two stars have almost the same filling factor.

For the error domain of the CaII mean equivalent width, we consider the solution for the lower value of the CaII mean equivalent width of $-0.82 \AA$. We also find a solution for the maximum value of $-1.56 \AA$. From these two solutions, we estimate the uncertainties in our model. We find that the resulting uncertainties in the model are $\delta \mathrm{H}_{\alpha}^{\mathrm{QR}}= \pm 0.009 \AA, \delta \mathrm{H}_{\alpha}^{\text {Plage }}= \pm 0.024 \AA$, $\delta \mathrm{CaII}^{\mathrm{QR}}= \pm 0.030 \AA, \delta \mathrm{CaII}^{\text {Plage }}= \pm 0.15 \AA, \delta f / f= \pm 18 \%$, $\delta \log (M)_{\mathrm{QR}}= \pm 0.023$, and $\delta \log (M)_{\text {Plage }}= \pm 0.019$. These figures are rather small if one considers the large uncertainty in the CaII mean equivalent width.

As far as the uncertainty in $\mathrm{H}_{\alpha}$ is concerned, we consider our solution for the CaII mean equivalent width of $-1.19 \AA$. We then consider the $\pm \sigma$ domain in $\mathrm{H}_{\alpha}$. We have two solutions for the upper value of $\mathrm{H}_{\alpha}$ of $0.487 \AA$. From these two solutions, we estimate the uncertainties in our model due to $\mathrm{H}_{\alpha}$. We find that the resulting uncertainties in the model are $\delta \mathrm{H}_{\alpha}^{\mathrm{QR}}= \pm 0.009 \AA, \delta \mathrm{H}_{\alpha}^{\text {Plage }}= \pm 0.094 \AA, \delta \mathrm{CaII}{ }^{\mathrm{QR}}= \pm 0.085 \AA$, $\delta \mathrm{CaII}^{\mathrm{Plage}}= \pm 0.43 \AA, \delta f / f= \pm 9 \%, \delta \log (M)_{\mathrm{QR}}= \pm 0.050$, and $\delta \log (M)_{\text {Plage }}= \pm 0.040$. We also list these values in Table 3 . These values are the smallest of our sample stars except for the filling factor. This is because the $\mathrm{H}_{\alpha}$ equivalent width value for our model is very close to the edge of the $\pm \sigma$ domain.

\section{Discussion}

We analyze in greater detail the differences between observations and models and see how they could help to improve the present models.

We obtain our best-fit model solutions for G1 205, GJ 1010A, and $\mathrm{Gl} 880$. Other models tend to produce $\mathrm{H}_{\alpha}$ profiles that are too strong in the wings, and as a result (because we reproduce the equivalent width) they also produce too deep $\mathrm{H}_{\alpha}$ profiles at line center. This is probably caused by blends in the wings of $\mathrm{H}_{\alpha}$. In Fig. 19, we show the $\mathrm{H}_{\alpha}$ profile for G1 205 with the spectral line identifications. One can see that there are blends in both wings of the $\mathrm{H}_{\alpha}$ profile: in the blue wing with a W I line, and in the red wing with Xe II, W I and Co I lines. One could argue that the W I and Xe II lines should be weak, although a careful analysis of this $\mathrm{H}_{\alpha}$ spectrum shows that in the vicinity of $\mathrm{H}_{\alpha}$ there are other W I, Hf II and Be I lines that are not so weak, in spite of the weak abundances of these elements. A careful analysis of a broader wavelength range shows that there are many pretty strong V I lines as well as other spectral lines from Sc I or Zr I (Paper X). Therefore, these blends might not be so weak, and detailed calculations will be necessary to derive firm conclusions.

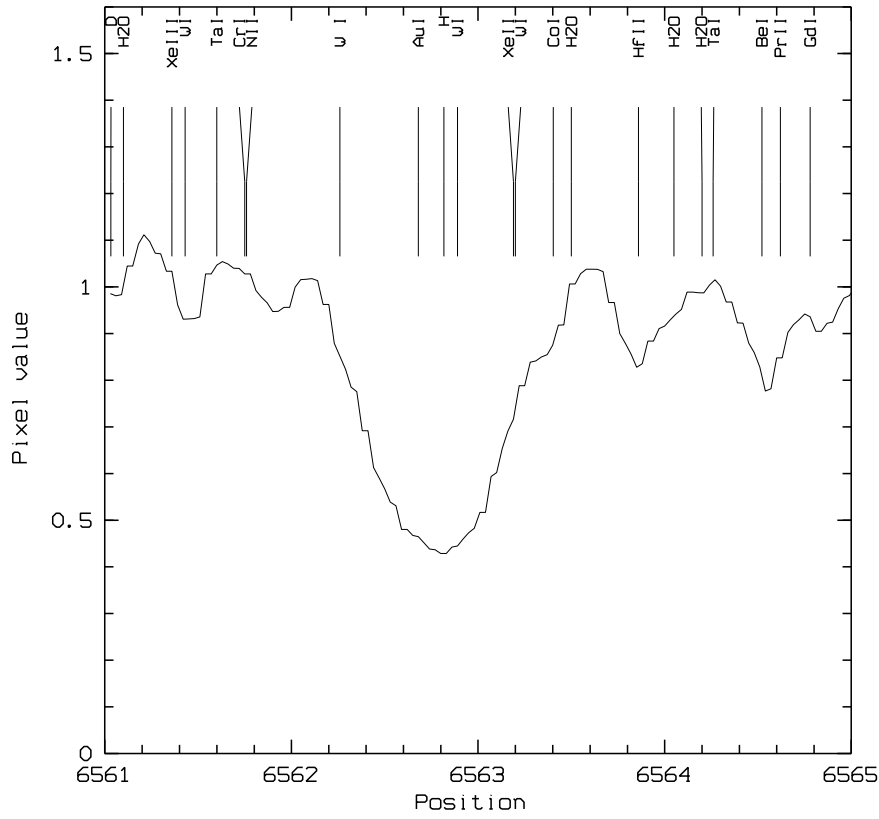

Fig. 19. We show the $\mathrm{H}_{\alpha}$ profile for Gl 205 with the spectral line identifications. One can see that there are blends in both wings of the $\mathrm{H}_{\alpha}$ profile. These blends tend to broaden the profile.

In this figure, we also include the telluric lines of $\mathrm{H}_{2} \mathrm{O}$ in the vicinity of $\mathrm{H}_{\alpha}$ from the solar spectrum of Beckers et al. (1976). One can see that there is also a blend with an $\mathrm{H}_{2} \mathrm{O}$ line in the red wing of $\mathrm{H}_{\alpha}$. These blends broaden the profile and seem to be responsible for the observed discrepancies between models and observations. We also note in Fig. 19 that $\mathrm{H}_{\alpha}$ is blended with both the Au I and W I lines at the line center. This may also alter the whole profile of $\mathrm{H}_{\alpha}$.

There is a systematic difference between the models and the observations in the $\mathrm{CaII} \mathrm{H/K}$ line ratio. Our models produce ratios that are too high, indicating that our model chromospheres are insufficiently optically thick. For the stars that we study here, this line ratio is very close to one, i.e., these lines are effectively optically thick in the chromosphere (see Paper VI). The difference between models and observations could be caused by underestimated temperature minima in our models. When the temperature minimum increases, the mass loading in the region of formation of the CaII lines indeed increases, and the optical thickness in these lines also increases. A rise in the temperature minimum also broadens the region of formation of the CaII lines and displaces this region of formation toward lower temperatures. However, this does not agree with the good agreement 
we have between models and observations for the minimum $\mathrm{H}_{\alpha}$ equivalent width we obtain for our models as a function of the mean CaII equivalent width.

Another systematic difference is in the CaII line centers: models produce profiles with a weak central absorption, whereas observations, albeit of a low $\mathrm{S} / \mathrm{N}$ ratio, show no or very weak central absorption. In our models (Paper VI), we took the solar values for micro-turbulence, i.e., $1 \mathrm{~km} \mathrm{~s}^{-1}$ at the temperature minimum, $2.5 \mathrm{~km} \mathrm{~s}^{-1}$ at the top of the chromosphere, and $4.6 \mathrm{~km} \mathrm{~s}^{-1}$ at the top of the atmosphere. More recent work shows that these values are probably underestimates. For instance, detailed work on $\epsilon$ Eridani by Sim \& Jordan (2003) shows that these values are about $7.5 \mathrm{~km} \mathrm{~s}^{-1}$ and $\sim 21 \mathrm{~km} \mathrm{~s}^{-1}$ at the top of the chromosphere and above $32000 \mathrm{~K}$ respectively. Pagano et al. (2000) analyzed STIS observations of AU Mic. They observed that the UV spectral lines have two components, a broad component and a narrow component. The narrow component dominates the flux in the spectral lines. They reported turbulent velocities typically in the range $10-25 \mathrm{~km} \mathrm{~s}^{-1}$ for neutral-element spectral lines (Si I, S I, C I, O I). These values are even higher than for $\epsilon$ Eridani. For higher temperature lines (C II, Si III, Si IV, $\mathrm{CIV}, \mathrm{NV}$ ), the turbulent velocities are typically in the range $25-35 \mathrm{~km} \mathrm{~s}^{-1}$ for the narrow component. For the broad component these values reach $100 \mathrm{~km} \mathrm{~s}^{-1}$ ! Therefore, although in dM1 stars, the turbulence is probably lower than in dM1e stars, our models probably underestimate the true turbulent velocities.

We find that our models correctly reproduce the FWHM of the $\mathrm{H}_{\alpha}$ and $\mathrm{Ca}$ II line profiles. However, we note that our theoretical CaII line profiles are systematically slightly narrower than the observed profiles. An increase in chromospheric turbulent velocities in the models would again provide a closer fit to the observations.

For the model uncertainties, we find that the uncertainties on $\mathrm{H}_{\alpha}$ and the column mass are the smallest, whereas those in $\mathrm{CaII}$ and the filling factor are larger. In particular, the uncertainties in the filling factor are always larger than $20 \%$ and may be as large as $60 \%$ ! This is an error that is intrinsic to our models. A finer grid of models would help reduce this error. The error in the column mass is typically $0.1 \mathrm{dex}$, which is fairly small. We can say that the model chromospheres are rather well constrained both for quiescent regions and active regions. In future observations, we hope to obtain a precision of $0.1 \AA$ for CaII and $0.01 \AA$ for $\mathrm{H}_{\alpha}$. This should yield a precision of about $10 \%$ for the filling factor. We cannot expect to obtain a higher precision in the filling factor.

Additional uncertainties may originate in the assumed shape of the model chromospheres of Houdebine \& Stempels (1997). Houdebine \& Doyle (1994a) found that the most accurate results for $\mathrm{dMe}$ stars are given by a linear temperature rise as a function of $\log (M)$. This result was also found for solar flare chromospheres (e.g., Hawley \& Fisher 1994), which have comparable atmospheric pressures. However, this may not be true for lower activity dM1 models, as is well known for instance for the Sun. Most authors (see references therein) have assumed a linear temperature rise, mostly for practical reasons. More work on the detailed modelling of individual stars is required to help progress in this direction. The disagreement between models and observations for the $\mathrm{CaII} \mathrm{H} / \mathrm{K}$ line ratio suggests that there is room for improvement in the model grid of chromospheres.

We show in Fig. 20 the minimum $\mathrm{H}_{\alpha}$ equivalent width that we obtain for our models, as a function of the mean CaII equivalent width. In other words, we cannot model stars that lie below this curve with the present grid of model

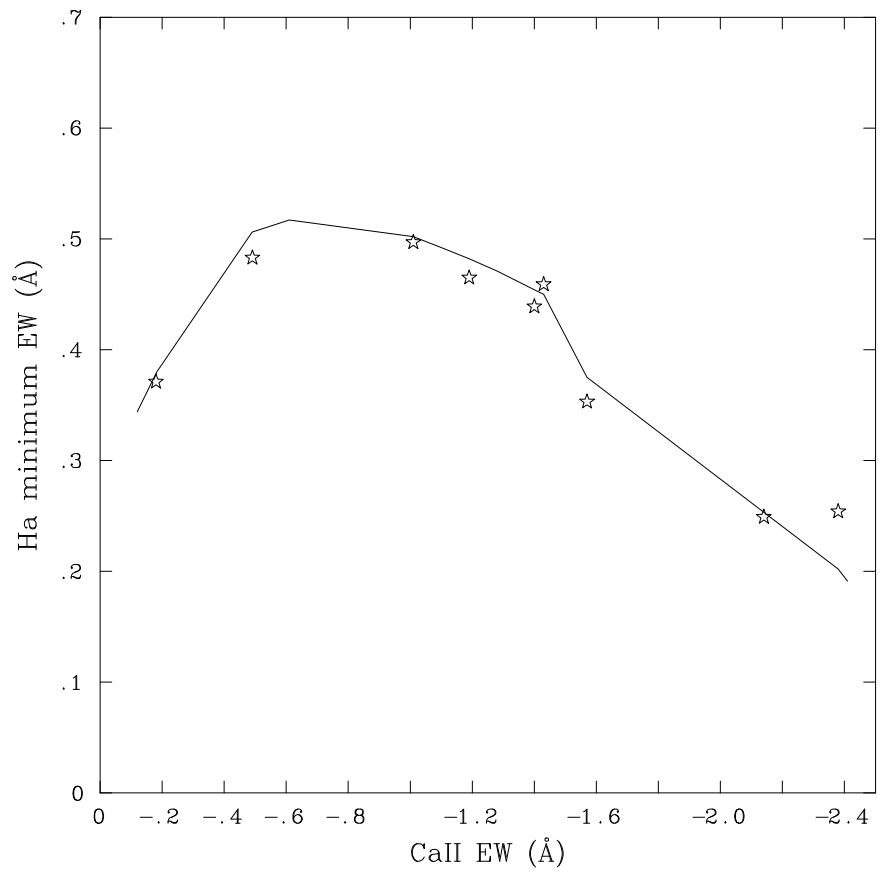

Fig. 20. We show the minimum $\mathrm{H}_{\alpha}$ observed equivalent width as a function of the mean $\mathrm{CaII} \mathrm{H} \& \mathrm{~K}$ equivalent width. The solid line represents the models, and the symbols the target stars investigated in this paper.

chromospheres. The shape of this curve is due to the unique variation in the $\mathrm{H}_{\alpha}$ equivalent width as a function of activity level for our grid of model chromospheres (see Paper VI). This minimum equivalent width decreases at low activity levels $\left(\mathrm{H}_{\alpha}\right.$ becomes weaker) and high activity levels when $\mathrm{H}_{\alpha}$ fills-in. We also plot our observations. One can see that observations delineate the same curve as the theoretical curve. We also note that because the observations lie close to the minimum theoretical curve, we then have solutions for all our stars within measurement errors. If we observe a star that is further below this curve, our models would not be able to reproduce the observations. Since there is good agreement between this theoretical curve and our observations, the validity of our models is strengthened.

We mentioned above that we possibly underestimated the temperature minima in our models of Paper VI. The good agreement that we find in Fig. 20 between models and observations, does not support this idea. Indeed, if we increase the temperature minima in the models, this would shift the theoretical curve towards higher CaII mean equivalent widths, and as a consequence the disagreement between observations and the theoretical curve would be larger at high activity levels. If we diminish the temperature minima, then the disagreement between observations and the theoretical curve would be larger at low activity levels. As a matter of fact, the present agreement is rather good, and further detailed modelling is required before we can obtain conclusions about the temperature minimum in our models.

Two stars have low activity levels (GJ 1010A and G1526). These latter stars are rare because according to the activityabsolute-magnitude correlation found in Paper VI, these rather large radius stars should have fairly high activity levels. We were surprised to find that for these two lowest activity stars, the plage filling factor is of the same order as for the other stars. The main consequence is that, it is not the plage filling factor that changes with the level of activity, but the chromospheric pressure that diminishes with the activity level. We illustrate this in Fig. 21, by plotting the column mass at the transition region as a function 


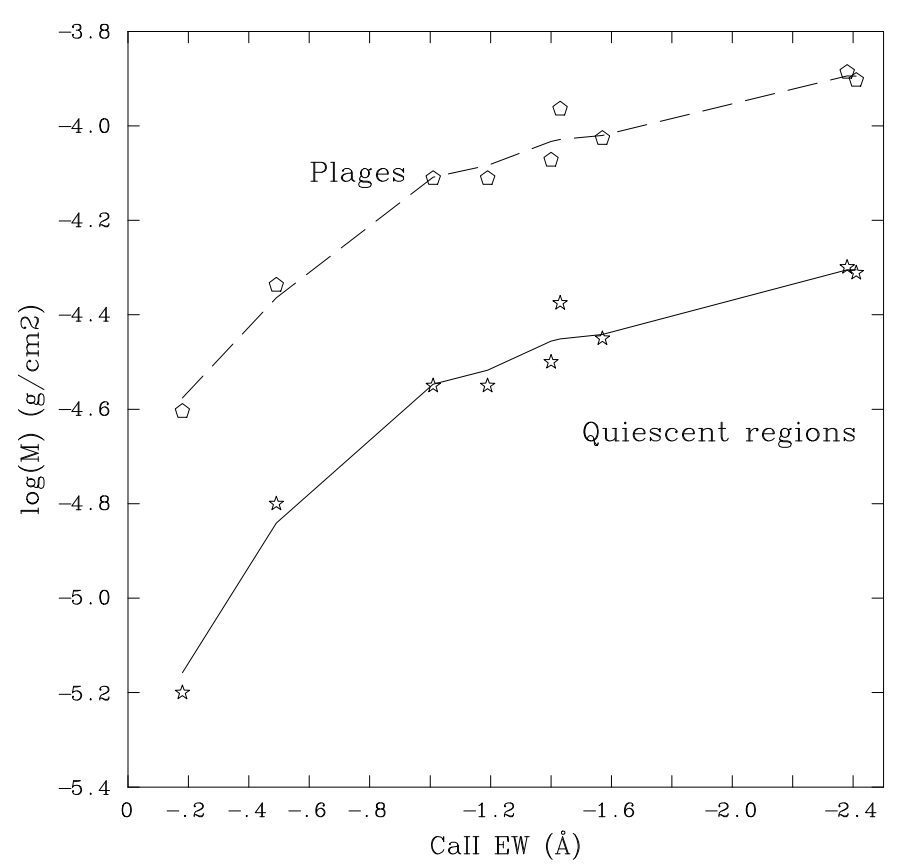

Fig. 21. We show the column mass (in $\mathrm{g} / \mathrm{cm}^{2}$ ) at about the transition region for active and quiescent regions for the nine dM1 stars that we studied, as a function of the observed mean CaII line equivalent width. There is a rather tight empirical correlation if one considers the large range in the activity covered by the observations.

of the mean CaII equivalent width. We find that both parameters correlate well for our sample stars. In other words, the chromospheric pressure is rather homogeneous for our sample stars, and it diminishes with the level of activity. This of course needs to be confirmed by additional studies, but it is promising to see that one could infer an estimate of the column mass at the transition region for both quiescent regions and active regions simply from the measurement of the mean CaII equivalent width. Similar results were obtained for the modelling of plages on the Sun (e.g., Shine \& Linsky 1974) for a similar pressure range than that of the dM1 stars studied here. Shine \& Linsky (1974) found that the CaII line core emission correlates well with the pressure at the top of the chromosphere, a parameter that is similar to the column mass at the transition region used here. This type of correlation is very interesting because it is relatively independent of the precise shape of the chromospheric temperature rise. However, this needs to be confirmed for dM1 stars by calculations of the effect of the temperature minimum on the CaII lines.

For the lowest activity star, GJ 1010A, the column masses at the transition region for quiescent regions and active regions are comparable to those found on the Sun. This in spite of the average surface flux of the CaII lines being significantly higher than for the Sun. This is because of the relatively large plage filling factor found for GJ 1010A. For all other stars, the column masses at the transition region are significantly higher than those found on the Sun. For the most active dM1 stars, G1 49 and G192-11A, the column mass for active regions is almost as high as those found on dM1e stars (e.g., AU Mic, Paper II).

Overall, we find that our two-component model chromospheres provide reasonable agreement with observations. For high activity dM1 stars such as G149 and G192-11A, onecomponent models could provide closer agreement with observations for the $\mathrm{H}_{\alpha}$ line, but the CaII line fluxes would then be largely underestimated. We cannot reproduce simultaneously the
$\mathrm{H}_{\alpha}$ and CaII equivalent widths with a single-component model chromosphere.

\section{Conclusion}

We have devised a new method of deriving two-component model chromospheres, based on simple constraints from the solar analogy and observations of active stars. We compared our models to observations of nine dM1 stars of near-solar metallicity. We found that our theoretical profiles agree reasonably well with observations of the $\mathrm{H}_{\alpha}$ line and the CaII resonance lines. Two stars have rather low activity levels, and for one of them (GJ 1010A) the theoretical models are comparable to those found for the Sun. The only difference is that for GJ 1010A, the plage filling factor is significantly higher than that of the Sun. We identified possible spectral line blends in the wings of the $\mathrm{H}_{\alpha}$ line. These blends seem to be responsible for the observed differences between models and observations. A comparison of theoretical and observed line profiles points to possible future improvements to our grid of model chromospheres. Notably, it seems that we have underestimated the turbulent velocities in the chromosphere and transition region.

We found plage filling factors in the range of $20 \%-40 \%$ for all our stars, even the least active. These values are comparable to those found for dMe stars. We also found a good agreement between the theoretical minimum $\mathrm{H}_{\alpha}$ equivalent width and the observations. This agreement provided further support to the validity of our grid of model chromospheres.

We obtained a tight correlation between the column mass at the transition region for quiescent and active regions, and the mean CaII equivalent width. This result is important if one considers that the CaII mean equivalent width varies by more than a factor of ten between low and high activity stars. This result is caused by the rather homogeneous plage filling factor that we find for all stars. As a consequence, it is the chromospheric pressure that increases with increasing activity level rather than the filling factor that increases. Although this finding needs to be confirmed, it is promising because the mean CaII equivalent width could be used to estimate directly the chromospheric pressure in quiescent and active regions alike.

Detailed modelling of basal chromospheres, active dM1e chromospheres, and intermediate activity dM1 chromospheres will be necessary to improve our grid of model chromospheres. Also, more high $\mathrm{S} / \mathrm{N}$ ratio, high resolution, observations are required to enable further progress in our modelling of $\mathrm{dM}$ star chromospheres.

Acknowledgements. The author would like to thank Prof. C.J. Butler from Armagh Observatory for improving the English of this manuscript, and the referee, Prof. J.L. Linsky for improving the content of this manuscript. The author would also like to thank Prof. M.S. Giampapa for useful comments. NSO/Kitt Peak FTS data used here were produced by NSF/NOAO.

\section{References}

Andretta, V., \& Giampapa, M. S. 1995, ApJ, 439, 405

Andretta, V., Doyle, J. G., \& Byrne, P. B. 1997, A\&A, 322, 266

Andretta, V., Jordan, S., Brosius, J. W., et al. 2000, ApJ 535, 438

Ayres, T. R., Marstad, N. C., \& Linsky, J. L. 1981, ApJ, 247, 545

Baliunas, S. L., Vaughan, A. H., Hartmann, L., et al. 1983, ApJ 275, 752

Beckers, J. M., Bridges, C. A., \& Gilliam, L. B. 1976, A High Resolution

Spectral Atlas of the Solar Irradiance From 380 to 700 Nanometers, AFGL-

TR-76-0126(I), Environmental Research Papers, No. 565

Chapman, G. A. 1980, ApJ 242, 45

Coluzzi, R. 1993, Bull. Inf. CDS, 43, 7 
Cram, L. E., \& Mullan, D. J. 1979, ApJ, 234, 579

Doyle, J. G., Houdebine, E. R., Mathioudakis, M., \& Panagi, P. 1994, A\&A, 285, 233

Fontenla, J. M., Avrett, E. H., \& Loeser, R. 2002, ApJ, 572, 636

Giampapa, M. S., Worden, S. P., Schneeberger, T. J., \& Cram, L. E. 1981, ApJ, 246, 502

Hawley, S. L., \& Fisher, G. H. 1994, ApJ, 426, 387

Houdebine, E. R. 1995, in Multi-site Continuous Spectroscopy, Proceedings of the 4th workshop held in Beijing, China, 179

Houdebine, E. R. 1996, in Stellar Surface Structures, ed. K. G. Strassmeier, \& J. L. Linsky, Proc. IAU Symp., 176, 547

Houdebine, E. R. 2008, MNRAS, 390, 1081 (Paper VII)

Houdebine, E. R. 2009a, PASP, submitted (Paper X)

Houdebine, E. R. 2009b, MNRAS, 397, 2133 (Paper XII)

Houdebine, E. R., \& Doyle, J. G. 1994a, A\&A, 289, 169 (Paper I)

Houdebine, E. R., \& Doyle, J. G. 1994b, A\&A, 289, 185 (Paper II)

Houdebine, E. R., \& Doyle, J. G. 1995a, A\&A, 302, 861 (Paper IV)

Houdebine, E. R., \& Doyle, J. G. 1995b, Irish Astr. J., 22, 25

Houdebine, E. R., \& Stempels, H. C. 1997, A\&A, 326, 1143 (Paper VI)

Houdebine, E. R., Doyle, J. G., \& Kościelecki, M. 1995, A\&A, 294, 773 (Paper III)

Houdebine, E. R., Mathioudakis, M., Doyle, J. G., \& Foing, B. H. 1996, A\&A, 305, 209 (Paper V)

Jevremovic, D., Doyle, J. G., \& Short, C. I. 2000, A\&A, 358, 575
Jordan, C. 1975, MNRAS, 170, 249

Jordan, C., Smith, G. R., \& Houdebine, E. R. 2005, MNRAS, 362, 411

Kelch, W. L., Linsky, J. L., \& Worden, S. P. 1979, ApJ, 229, 700

Lanzafame, A. C., \& Byrne, P. B. 1995, A\&A, 303, 155

Leggett, S. K. 1992, ApJS, 82, 351

Mauas, P. J. D. 2000, ApJ, 539, 858

Mauas, P. J. D., \& Falchi, A. 1994, A\&A, 281, 129

Neff, J. E., Walter, F. M., Rodono, M., \& Linsky, J. L. 1989, A\&A, 215, 79

Pagano, I., Linsky, J. L., Carkner, L., et al. 2000, ApJ, 532, 497

Pagano, I., Rodono, M., Linsky, J. L., et al. 2001, A\&A, 365, 128

Queloz, D. 1994, Internal Publication of the Observatoire de Haute Provence:

ELODIE, Programme de Réduction, OHP, St Michel l'Observatoire, France.

Shine, R. A., \& Linsky, J. L. 1974, Sol. Phys., 39, 49

Short, C. I., \& Doyle, J. G. 1998a, A\&A, 331, L5

Short, C. I., \& Doyle, J. G. 1998b, A\&A, 336, 613

Short, C. I., Doyle, J. G., \& Byrne, P. B. 1997, A\&A, 324, 196

Sim, S. A., \& Jordan, C. 2003, MNRAS, 341, 517

Smith, G. R. 2003, MNRAS, 341, 143

Smith, G. R., Houdebine, E. R., \& Jordan, C. 2009, in preparation

Vaughan, A. H., Baliunas, S. L., Middelkoop, F., et al. 1981, ApJ, 250, 276

Walkowicz, L. M. 2008, Ph.D. Thesis dissertation of the University of Washington, Self-consistent quiescent model atmospheres for M dwarfs

Walter, F. M., Neff, J. E., Gibson, D. M., Linsky, J. L., \& Rodono, M. 1987, A\&A, 186, 241 